\title{
Association between Dietary Fibre Intake and Colorectal Adenoma: A Systematic Review and Meta-Analysis
}

\author{
Daniele Nucci $^{1}{ }^{1}$, Cristina Fatigoni ${ }^{2}\left(\mathbb{D}\right.$, Tania Salvatori ${ }^{2}$, Mariateresa Nardi ${ }^{1}$, Stefano Realdon ${ }^{3}$ \\ and Vincenza Gianfredi $4,5, *$ (D)
}

1 Nutritional Support Unit, Veneto Institute of Oncology IOV-IRCCS, via Gattamelata 64, 35128 Padua, Italy; daniele.nucci@iov.veneto.it (D.N.); mariateresa.nardi@iov.veneto.it (M.N.)

2 Department of Pharmaceutical Sciences, University of Perugia, via del Giochetto, 06122 Perugia, Italy; cristina.fatigoni@unipg.it (C.F.); tania.salvatori90@gmail.com (T.S.)

3 Digestive Endoscopy Unit, Veneto Institute of Oncology IOV-IRCCS, via Gattamelata 64, 35128 Padua, Italy; stefano.realdon@iov.veneto.it

4 School of Medicine, University Vita-Salute San Raffaele, 20132 Milan, Italy

5 CAPHRI Care and Public Health Research Institute, Maastricht University, 6211 Maastricht, The Netherlands

* Correspondence: gianfredi.vincenza@hsr.it

check for

updates

Citation: Nucci, D.; Fatigoni, C.; Salvatori, T.; Nardi, M.; Realdon, S.; Gianfredi, V. Association between Dietary Fibre Intake and Colorectal Adenoma: A Systematic Review and Meta-Analysis. Int. J. Environ. Res. Public Health 2021, 18, 4168. https://doi.org/10.3390/ ijerph18084168

Academic Editor: Paul B. Tchounwou

Received: 3 March 2021

Accepted: 13 April 2021

Published: 15 April 2021

Publisher's Note: MDPI stays neutral with regard to jurisdictional claims in published maps and institutional affiliations.

Copyright: (c) 2021 by the authors. Licensee MDPI, Basel, Switzerland. This article is an open access article distributed under the terms and conditions of the Creative Commons Attribution (CC BY) license (https:// creativecommons.org/licenses/by/ $4.0 /)$.

\begin{abstract}
PubMed/Medline, Excerpta Medica dataBASE (EMBASE) and Scopus were searched in January 2021 in order to retrieve evidence assessing the association between dietary fibre intake and the risk of colorectal adenoma in adults. Preferred Reporting Items for Systematic Reviews and Meta-Analyses (PRISMA) guidelines were used for the reporting of results. Only primary observational studies were included. Publication bias was estimated through the Egger's test and the visual inspection of the funnel plot. Heterogeneity between studies was calculated with $\mathrm{I}^{2}$ statistics. The search strategy identified 683 papers, 21 of which were included in our meta-analysis. Having evaluated a total of 157,725 subjects, the results suggest a protective effect of dietary fibre intake against colorectal adenoma. Effect Size (ES) was [0.71 (95\% CI $=0.68-0.75), p=0.000)]$. Moderate statistical heterogeneity $\left(\mathrm{Chi}^{2}=61.68, \mathrm{df}=23, \mathrm{I}^{2}=62.71 \%, p=0.000\right)$ was found. Findings show a statistically significant $(p=0.000)$ and robust association between a higher intake of dietary fibre and a lower risk of colorectal adenoma, considering both the prevalent and incident risk. Moreover, the meta-regression analysis showed a borderline significant negative linear correlation between the amount of dietary fibre intake and colorectal adenoma. Lastly, we performed a subgroup analysis by sex, showing a higher protective effect for men.
\end{abstract}

Keywords: diet; fibre; colorectal; adenoma; systematic review

\section{Introduction}

Colorectal cancer is the third most common cancer among men (after lung and prostate cancer) and the second among women (after breast cancer) worldwide, with approximately 2 new million cases (among both men and women) in 2020 [1]. Colorectal cancer is one of the few cancers for which a population screening program is in place practically all over the world [2]. There are several important reasons why colorectal cancer is suitable for population screening, including cancer progression from a preneoplastic (and subclinical) lesion (adenoma), the long lag time before invasive and malignant transformation, an easily detectable and treatable preneoplastic lesion, and the direct association between the stage of the disease and mortality [3]. It should be noted that colorectal adenoma is a proliferative dysplastic epithelial lesion that is harmful in most cases. It can have a malignant evolution based on the size, number, histology (grade of dysplasia) and duration in time [4]. Moreover, some other unmodifiable and modifiable factors might play an important role, such as age, ethnicity and genetics. Smoking, body mass index and diet seem to be the most important modifiable risk factors [5]. A high-fibre diet provide several 
plausible biological mechanisms that potentially provide a beneficial effect. Fibre might play a protective role through several mechanisms, including physical mechanisms, antiinflammatory properties and prebiotic effects. Results from two extensive and recent metaanalyses confirm the protective role of fibre on colon [6] and rectal cancer risk [7]. However, despite the fact that adenoma as a preneoplastic lesion is recognized as a precursor of colorectal cancer, previous studies failed to univocally assess the role of dietary fibre intake and the risk of colorectal adenoma. These inconclusive results are probably due to a small population size, differences in the population's characteristics, the adenoma site, the follow-up duration or the dose of fibre intake.

In this perspective, we performed the current systematic review with meta-analysis in order to collect and retrieve all relevant studies assessing the association between dietary fibre intake and the risk of colorectal adenoma. Moreover, we aimed to identify the amount of dietary fibre useful to prevent colorectal adenoma and also to estimate the risk among women and men and to evaluate the different risks of incident and prevalent colorectal adenoma.

\section{Materials and Methods}

The current systematic review with meta-analysis was accomplished following the methods recommended by the Cochrane Collaboration [8] and the Meta-analysis Of Observational Studies in Epidemiology (MOOSE) guidelines [9]. We followed the Preferred Reporting Items for Systematic Reviews and Meta-Analyses (PRISMA) [10] guidelines [11] to report the process and results. The review protocol was registered, in advance, on the International Prospective Register of Systematic Reviews (PROSPERO) (ID number: CRD42021230276), funded by the National Institute of Health Research (https://www.crd.york.ac.uk/prospero/, accessed on 8 January 2021). This systematic review was developed to answer the following research question: "Is dietary fibre intake associated with a lower (or higher) risk of colorectal adenoma?"

\subsection{Search Strategy and Data Sources}

Searches were independently carried out on the PubMed/Medline, Excerpta Medica dataBASE (EMBASE) and Scopus databases by two of the authors (VG and DN) in order to identify eligible studies. The structured computer literature search was built on a pre-determined combination of keywords, according to the type of database consulted. The search strategy was developed based on three domains: dietary fibre intake, colorectal adenoma and study design. Selected keywords were opportunely combined using Boolean operators. Moreover, we also built our search strategy using Medical Subject Headings (MeSH), text and title/abstract words. The strategy was first developed in PubMed/MEDLINE and then adapted for use in the other databases. The full search strategy is reported in Supplementary Table S1. The literature search on all the databases was carried out on the same day (10 January 2021) and included articles from inception (no time filter was used). Reference lists were also screened in order to identify additional relevant articles and experts in the field were consulted. The corresponding authors of included articles were contacted in case of missing data.

\subsection{Inclusion/Exclusion Criteria}

As recommended by the Cochrane Collaboration [12], a detailed description of inclusion/exclusion criteria, based on a Population, Exposure, Comparison, Outcomes and Study (PECOS) design [13], is reported in Supplementary Table S2. In brief, only those articles assessing the association between dietary fibre intake in healthy adults (both men and women) and the risk of colorectal adenoma were considered eligible. On the contrary, those studies that were conducted to assess the different outcomes or different fibre intake (for instance, supplementation) among subjects with comorbidities were excluded. With reference to the study design, only observational studies (cohort, case-control, cross-sectional or ecological studies) were considered for eligibility. Outcomes should be reported as risks 
[e.g., Odds Ratio (OR), Risk Ratio (RR) or Hazard Ratio (HR)], with their 95\% Confidence Interval (CI 95\%), or as a mean. Lastly, only articles published in English and with full-text available were considered.

\subsection{Data Extraction}

As had been done in previous studies [14,15], a two-step double-blind selection process was adopted by two researchers (DN and VG) to identify relevant articles. The selection process was firstly based on title and abstract screening; therefore the full-text was only obtained for potentially relevant studies. Data screening and data extraction was independently performed by two researchers (DN and CF) using a spreadsheet created in Microsoft Excel ${ }^{\circledR}$ for Windows. The spreadsheet was pre-piloted on 5 randomly selected papers. This allowed us to increase methodological concordance. Selected articles and extracted data were compared, with any discrepancies being resolved throught discussion between the two researchers ( $\mathrm{DN}$ and $\mathrm{CF}$ ). If the disagreement persisted, a third researcher was consulted (VG). Both qualitative and quantitative data were extracted. Recorded quantitative data included sample size, study duration (expressed in years), amount of fibre intake and outcome (expressed as risk). Any adjusted estimated risks available in primary studies, were used preferentially. Qualitative data included: name of the first author, year of publication, country where the study was conducted, subjects' characteristics, outcome measured, and tools used to assess dietary information and outcome diagnosis. Moreover, the presence of funding supporting the original research studies and information on conflict of interests (CoI) were also recorded.

\subsection{Critical Appraisal}

Two researchers (CF and DN) independently performed the critical appraisal, using the Newcastle-Ottawa Scale (NOS) [16] for observational studies. However, since the NOS did not provide a checklist for cross-sectional studies, we used a modified version [17] adapted to perform a quality assessment of cross-sectional studies. Using these criteria and based on a standard cut-off, we identified high-quality studies by means of the NOS if this was equal to or higher than 7 points.

\subsection{Statistical Analysis}

The effect size (ES) was calculated based on the odds ratio (OR), risk ratio (RR) and mean and sample size provided per study. It was estimated by OR and reported with its $95 \%$ CI. If papers presented a number of events (cases) among those exposed and not exposed and the mean value for dietary fibre intake for each group, the OR and CIs were computed from these data and included in the meta-analysis. The comparison was performed between subjects exposed to the highest intake of dietary fibre and subjects exposed to the lowest (or none) intake of dietary fibre consumption. Since the dietary fibre intake collected in each study was homogeneously reported, it allowed us to perform a meta-regression analysis. We applied a fixed and random model. A fixed model is used when the universe of studies is judged a priori to be sufficiently similar to those in the study sample, or when the number of included studies is small. The assumption behind the random effect model is that inferences are not limited to studies represented in the sample. In other words, it is presumed that the universe of studies contains studies with differences in their characteristics, and generalizations are based on studies that differ from those in the study sample. In this perspective, the random effect model is recommended if heterogeneity estimated values are considered high. Heterogeneity among included studies was evaluated through $\mathrm{Chi}^{2}$ and $\mathrm{I}^{2}$ tests. Heterogeneity was considered to be high when $\mathrm{I}^{2}$ values $>75 \%$, moderate for $\mathrm{I}^{2}$ values ranging between $50 \%$ and $75 \%$, low for values ranging between $25 \%$ and $50 \%$, and no heterogeneity for values below $25 \%$. The graphical evaluation of the Funnel plot and the Egger's regression asymmetry test were used to estimate potential publication bias; statistical significance was set at $p<0.10$ [18]. If any publication bias was detected, a trim and fill method, to search for missing studies to the 
right of overall, was used in order to adjust for publication bias [19]. The meta-analysis was performed using the Prometa ${ }^{\circledR}$ (Internovi, Cesena, Italy) software.

\subsection{Sub-Group and Sensitivity Analysis}

In order to exclude a potential overlapping effect due to the inclusion of studies referring to the same cohort of patients, a sensitivity analysis was run which only considered the study with the highest quality score (QS) or with the larger sample size in case of equal QS. We also performed the meta-analysis by excluding studies with computed OR. Furthermore, sensitivity analyses were conducted which only included studies with a follow-up (FU) equal to or higher than 9 years, with validated tools to assess dietary fibre intake, the type of diagnosis and a QS higher than 7. In addition, in order to corroborate the obtained results, sub-group analyses were performed based on the adenoma site, study design (case-control and cross-sectional vs. cohort studies) and sex.

\subsection{Cumulative Analysis}

A cumulative analysis is a sequential meta-analysis, intended to evaluate how adding one study at a time modifies the ES. We performed three cumulative analyses: the first one adding studies chronologically (starting from the first published analysis to the most recent publication), the second one based on the growing sample size (from the smallest to the biggest), and the third based on the ascending dose of dietary fibre intake (from the lowest to the highest). These types of analyses improve the potential consistency of results [20].

\section{Results}

\subsection{Literature Search}

We identified a total of 683 articles as follows: 424 in PubMed/Medline, 204 in the Scopus and 55 in EMBASE. After the removal of duplicates, a total of 569 original articles were preliminarily screened by title and abstract. From these, 549 were excluded because they were unrelated topics $(n=443)$, reviews $(n=55)$, not original studies (erratum, conference paper, commentary, letter and book chapters $n=21)$, in a different language $(n=19)$, in vitro studies $(n=8)$ and in vivo studies $(n=4)$. Overall, 29 studies were eligible, but 8 studies were excluded because of the reasons reported in Supplementary Table S2 [21-28]. Figure 1 depicts the flow diagram reporting the selection process. At the end of the screening process, 21 articles were included in the quantitative analysis [29-49]; however, because four papers reported separate data based on the adenoma site (colorectal, colon and rectal) $[29,37,44,45]$, one paper reported the results for both incident and recurrent adenoma [37], another used a different control group (general population and hospitalbased patients) [29], and two papers reported data stratified by sex [36,46], these were considered to be independent studies. Lastly, five studies [30,35,36,45,49] did not report the association between dietary fibre intake and colorectal adenoma as a risk, but as a number of events among those with higher and lower intake. Thus, ORs were computed for this reason. We considered them in the overall pooled estimate, but removed them in the sensitivity analysis. 


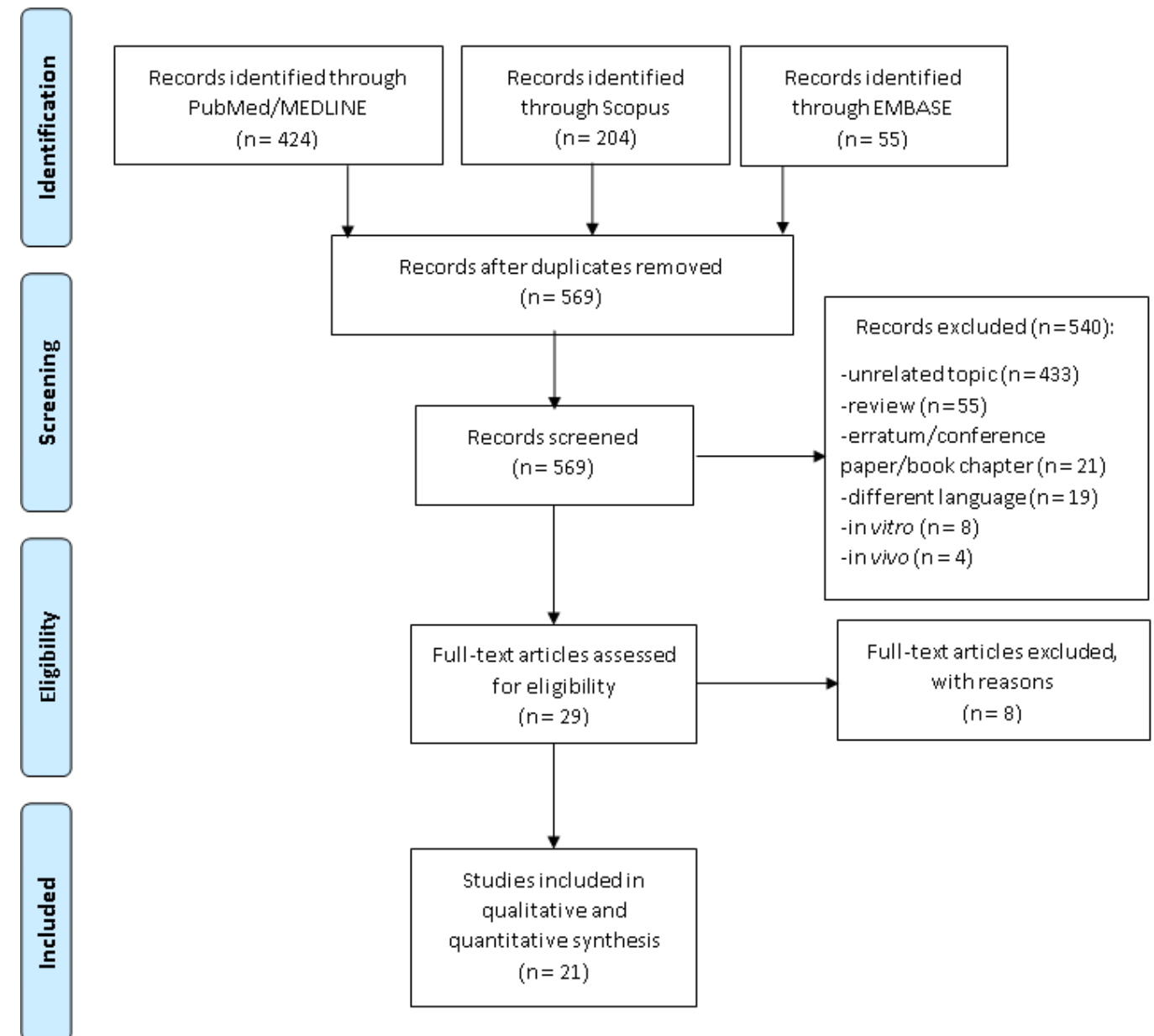

Figure 1. Flow diagram of the studies' selection process.

\subsection{Characteristics of Included Studies}

Tables 1 and 2 show the characteristics of the studies included in the meta-analysis in alphabetical order. Supplementary Table S3 shows the quality evaluation. The first included study was published in 1986 [36], whereas the most recent was published in 2020 [30], however, the period with the highest number of publications on the topic were the decades 1990-2000 and 2010-2020. Almost all the included studies were conducted in the United States of America (USA), but one was conducted in Israel [39] and two in Europe (Germany [29] and Norway [36], respectively). As regards the study design, 13 studies were case-control [29-31,34,36,38-42,44,46,49], seven were cohort studies [32,33,35,37,43,45,48], and one was a cross-sectional study [47]. Only considering cohort studies, the follow-up (FU) period ranged between 2 years to 26 years [43]. The sample size ranged between 100 [36] and 37,562 participants [44], with a population age ranging from 18 to 79 years. More than half of the included studies $(n=13)$ used a validated self- administered Food Frequency Questionnaire (FFQ) to assess dietary fibre intake [30-35,37,41-45,49], one study used a diet history questionnaire [47], and another used the food diary [36]. Furthermore, five studies performed an interview [29,38-40,46], however three studies did not provide information on validation $[40,44,48]$. When pooling data in meta-analysis, higher dietary fibre intake was associated with a lower risk of colorectal adenoma [in the fixed effect model, pooled ES $=0.71(95 \% \mathrm{CI}=0.68-0.75), p=0.000$; in the random effect model, pooled $\mathrm{ES}=0.73(95 \% \mathrm{CI}=0.66-0.81), p=0.000$; based on 157,725 participants, with moderate statistical heterogeneity $\left(\mathrm{Chi}^{2}=61.68, \mathrm{df}=23, \mathrm{I}^{2}=62.71 \%, p=0.000\right)$ ] (Figure 2a) and potential publication bias (Figure 2b), at which trim and fill method was applied (Table 3). 
Table 1. Qualitative characteristics of included studies, reported in alphabetical order.

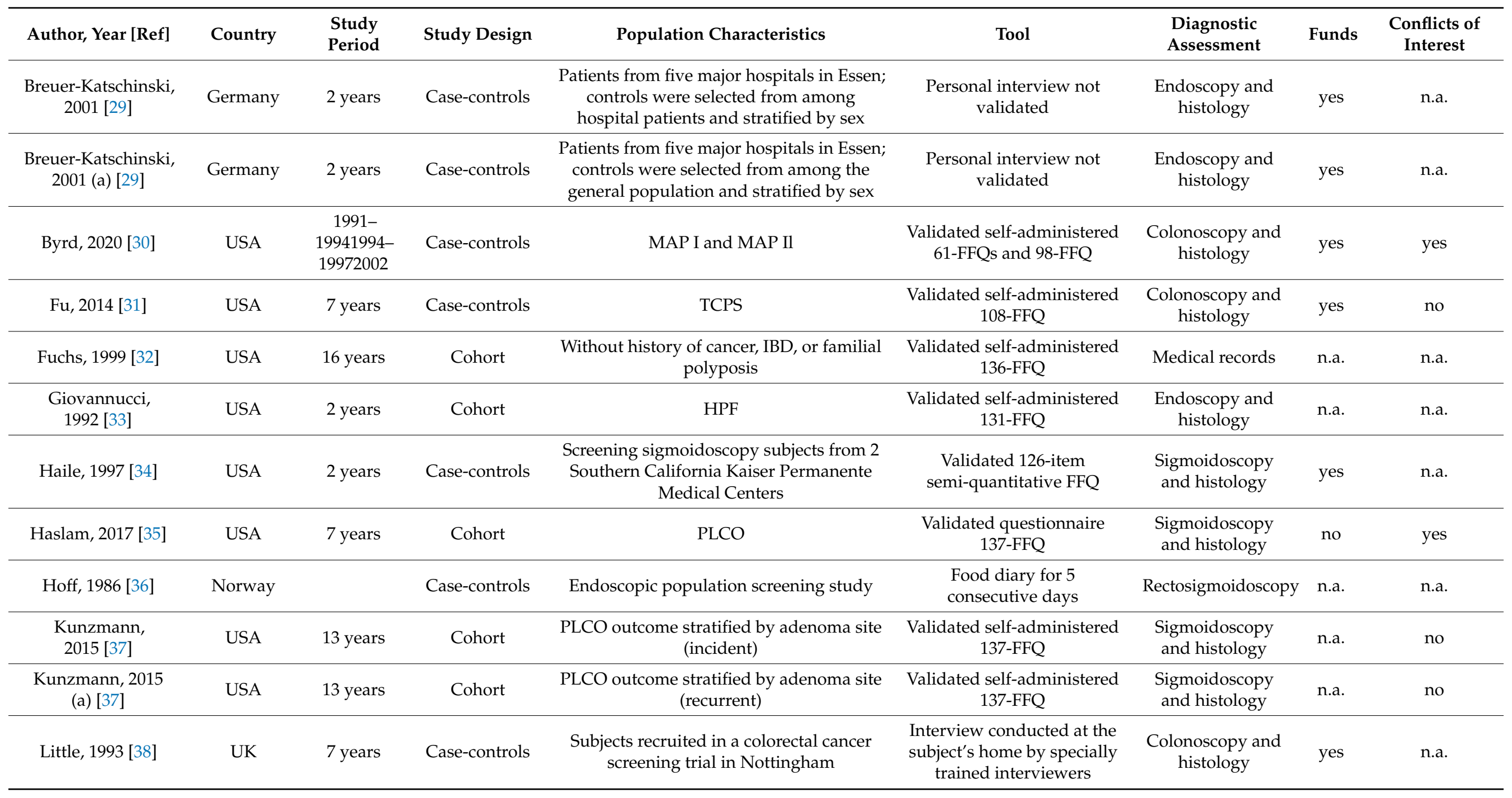


Table 1. Cont.

\begin{tabular}{|c|c|c|c|c|c|c|c|c|}
\hline Author, Year [Ref] & Country & $\begin{array}{l}\text { Study } \\
\text { Period }\end{array}$ & Study Design & Population Characteristics & Tool & $\begin{array}{c}\text { Diagnostic } \\
\text { Assessment }\end{array}$ & Funds & $\begin{array}{l}\text { Conflicts of } \\
\text { Interest }\end{array}$ \\
\hline Lubin, 1997 [39] & Israel & 3 years & $\begin{array}{c}\text { Paired } \\
\text { Case-controls }\end{array}$ & $\begin{array}{l}\text { Subjects identified in the SPGD at the Tel } \\
\text { Aviv Medical Center }\end{array}$ & $\begin{array}{l}\text { 180-item questionnaire } \\
\text { (interview) }\end{array}$ & $\begin{array}{l}\text { Endoscopy and } \\
\text { histology }\end{array}$ & n.a. & n.a. \\
\hline Martìnez, 1996 [40] & USA & 2 years & Case-controls & $\begin{array}{l}\text { Population without history of colorectal } \\
\text { polyps and familial polyposis }\end{array}$ & $\begin{array}{l}\text { 138-FFQ (interview) } \\
\text { validation n.a. }\end{array}$ & $\begin{array}{l}\text { Sigmoidoscopy or } \\
\text { colonoscopy and } \\
\text { histology }\end{array}$ & n.a. & n.a. \\
\hline Mathew, 2004 [41] & USA & 2 years & Case-controls & $\begin{array}{l}\text { Subjects with new or recurrent adenomas } \\
\text { in a study conducted at the NNMC }\end{array}$ & $\begin{array}{l}\text { Validated self-administered } \\
\text { 100-item }\end{array}$ & $\begin{array}{l}\text { Sigmoidoscopy or } \\
\text { colonoscopy and } \\
\text { histology }\end{array}$ & n.a. & n.a. \\
\hline Mujtaba,2018 [42] & USA & $\begin{array}{c}1991- \\
19941994- \\
19972002\end{array}$ & Case-controls & $\begin{array}{l}\text { CPRU } \\
\text { MAP I } \\
\text { MAP Il }\end{array}$ & $\begin{array}{l}\text { Validated self-administered } \\
\text { 61-FFQs }\end{array}$ & $\begin{array}{l}\text { Colonoscopy and } \\
\text { histology }\end{array}$ & n.a. & no \\
\hline Nimptsch, 2014 [43] & USA & 9 years & Cohort & NHS II & $\begin{array}{l}\text { Validated self-administered } \\
\text { 131-FFQ }\end{array}$ & Medical record & yes & n.a. \\
\hline Peters, 2003 [44] & USA & 7 years & Case-controls & PLCO & $\begin{array}{c}\text { Self-administered 137-FFQ } \\
\text { (adaptation from previous } \\
\text { validated FFQ) }\end{array}$ & $\begin{array}{l}\text { Endoscopy and } \\
\text { histology }\end{array}$ & n.a. & n.a. \\
\hline Platz, 1997 [45] & USA & 8years & Cohort & $\mathrm{HPF}$ & $\begin{array}{l}\text { Validated self-administered } \\
\text { 131-FFQ) }\end{array}$ & $\begin{array}{l}\text { Sigmoidoscopy or } \\
\text { colonoscopy and } \\
\text { histology }\end{array}$ & yes & n.a. \\
\hline Sandler, 1993 [46] & USA & 2 years & Case-controls & $\begin{array}{l}\text { Subjects who underwent colonoscopy at } \\
\text { the University of North Carolina Hospitals }\end{array}$ & $\begin{array}{l}\text { Validated quantitative food } \\
\text { frequency questionnaire } \\
\text { (interview) }\end{array}$ & $\begin{array}{l}\text { Sigmoidoscopy or } \\
\text { colonoscopy and } \\
\text { histology }\end{array}$ & n.a. & n.a. \\
\hline Shaw, 2017 [47] & USA & 7 years & $\begin{array}{c}\text { Cross- } \\
\text { sectional }\end{array}$ & FMCCSC & DHQ I or II & $\begin{array}{l}\text { Colonoscopy and } \\
\text { histology }\end{array}$ & yes & no \\
\hline $\begin{array}{l}\text { Tantamango, } \\
2011[48]\end{array}$ & USA & 26 years & Cohort & AHS- 1 and AHS-2 & $\begin{array}{l}\text { Self-administered 55-FFQ } \\
\text { validation n.a. }\end{array}$ & Self-reported & yes & no \\
\hline Witte, 1996 [49] & USA & 2 years & $\begin{array}{c}\text { Paired } \\
\text { Case-controls }\end{array}$ & $\begin{array}{l}\text { Subjects free of invasive cancer, IBD and } \\
\text { familial polyposis }\end{array}$ & $\begin{array}{l}\text { Validated self-administered } \\
126-\mathrm{FFQ}\end{array}$ & $\begin{array}{l}\text { Sigmoidoscopy } \\
\text { and histology }\end{array}$ & n.a. & n.a. \\
\hline
\end{tabular}

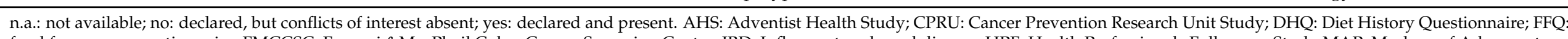

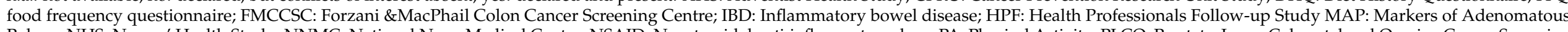

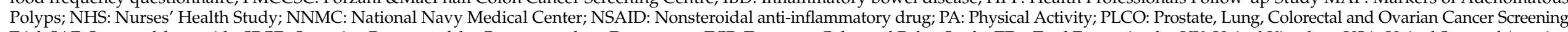

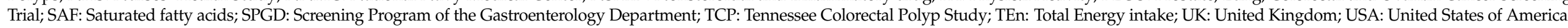


Table 2. Quantitative characteristics of included studies, reported in alphabetical order.

\begin{tabular}{|c|c|c|c|c|c|c|c|c|c|}
\hline $\begin{array}{c}\text { Author, Year [Ref] } \\
\text { (Number of Stratified } \\
\text { Analysis) }\end{array}$ & $\begin{array}{c}\text { Total } \\
\text { Sample }\end{array}$ & Sex & $\begin{array}{l}\text { Age (In Years) } \\
\text { Mean } \pm \text { SD }\end{array}$ & $\begin{array}{c}\text { Dietary Fibre } \\
\text { Intake Mean } \pm \text { SD }\end{array}$ & Outcome & $\begin{array}{l}\text { n. Subjects at } \\
\text { the Highest } \\
\text { Fibre Intake }\end{array}$ & $\begin{array}{l}\text { Highest Dietary } \\
\text { Fibre Intake }\end{array}$ & $\begin{array}{c}\text { Effect Size }(95 \% \text { CI }) \\
p\end{array}$ & Adjustment \\
\hline $\begin{array}{l}\text { Breuer-Katschinski, } \\
2001 \text { [29] (1) }\end{array}$ & $\begin{array}{l}\text { Ca: } 182 \\
\text { Co I: } 178\end{array}$ & $\begin{array}{l}\text { Ca: } \mathrm{M}=94 \\
\text { Co I: } \mathrm{M}=88\end{array}$ & $\begin{array}{l}\text { Ca: } 63.8 \pm 9.9 \\
\text { Co I: } 63.4 \pm 9.8\end{array}$ & $\begin{array}{l}\text { Ca: } 23.01 \pm 7.68 \mathrm{~g} / \mathrm{d} \\
\text { Co: } 24.27 \pm 8.09 \mathrm{~g} / \mathrm{d}\end{array}$ & \multirow{3}{*}{$\begin{array}{l}\text { colorectal } \\
\text { adenoma }\end{array}$} & \multirow{3}{*}{ n.a. } & \multirow{3}{*}{ n.a. } & $\begin{array}{c}\text { RR } 0.47 \\
(0.23-0.99) \\
p<0.05\end{array}$ & \multirow{3}{*}{ TEn, BMI, and social class } \\
\hline $\begin{array}{l}\text { Breuer-Katschinski, } \\
2001[29](2)\end{array}$ & $\begin{array}{l}\text { Ca: } 94 \\
\text { Co I: } 88\end{array}$ & $\begin{array}{c}\text { Ca: } M=94 \\
\text { Co I: } M=88\end{array}$ & $\begin{array}{l}\text { Ca: } 63.8 \pm 9.9 \\
\text { Co I: } 63.4 \pm 9.8\end{array}$ & n.a. & & & & $\begin{array}{c}\text { RR } 0.16 \\
(0.05-0.57) \\
p<0.05\end{array}$ & \\
\hline $\begin{array}{l}\text { Breuer-Katschinski, } \\
2001[29] \text { (3) }\end{array}$ & $\begin{array}{l}\text { Ca: } 88 \\
\text { Co I: } 90\end{array}$ & $\begin{array}{l}\text { Ca: } F=88 \\
\text { Co I: } F=90\end{array}$ & $\begin{array}{l}\text { Ca: } 63.8 \pm 9.9 \\
\text { Co I: } 63.4 \pm 9.8\end{array}$ & n.a. & & & & $\begin{array}{c}\text { RR } 0.66 \\
(0.23-1.86) \\
p=\text { n.s. }\end{array}$ & \\
\hline $\begin{array}{l}\text { Breuer-Katschinski, } \\
2001 \text { (a) [29] (4) }\end{array}$ & $\begin{array}{l}\text { Ca: } 182 \\
\text { Co II: } 182\end{array}$ & $\begin{array}{c}\text { Ca: } \mathrm{M}=94 \\
\text { Co II: } \mathrm{M}=92\end{array}$ & $\begin{array}{c}\text { Ca: } 63.8 \pm 9.9 \\
\text { Co II: } 64.2 \pm 9.9\end{array}$ & $\begin{array}{l}\text { Ca: } 23.01 \pm 7.68 \mathrm{~g} / \mathrm{d} \\
\text { Co: } 23.60 \pm 6.75 \mathrm{~g} / \mathrm{d}\end{array}$ & \multirow{3}{*}{$\begin{array}{l}\text { colorectal } \\
\text { adenoma }\end{array}$} & \multirow{3}{*}{ n.a. } & \multirow{3}{*}{ n.a. } & $\begin{array}{c}\text { RR } 0.87 \\
(0.33-2.33) \\
p=\text { n.s. }\end{array}$ & \multirow{3}{*}{ TEn, BMI, and social class } \\
\hline $\begin{array}{l}\text { Breuer-Katschinski, } \\
2001 \text { (a) [29] (5) }\end{array}$ & $\begin{array}{l}\text { Ca: } 94 \\
\text { Co II: } 92\end{array}$ & $\begin{array}{c}\text { Ca: } \mathrm{M}=94 \\
\text { Co II: } \mathrm{M}=92\end{array}$ & $\begin{array}{c}\text { Ca: } 63.8 \pm 9.9 \\
\text { Co II: } 64.2 \pm 9.9\end{array}$ & n.a. & & & & $\begin{array}{l}\text { RR } 0.38 \\
(0.14-1.05) \\
p=\text { n.s. }\end{array}$ & \\
\hline $\begin{array}{l}\text { Breuer-Katschinski, } \\
2001 \text { (a) [29] (6) }\end{array}$ & $\begin{array}{l}\text { Ca: } 88 \\
\text { Co II: } 90\end{array}$ & $\begin{array}{c}\text { Ca: } F=88 \\
\text { Co II: } F=90\end{array}$ & $\begin{array}{c}\text { Ca: } 63.8 \pm 9.9 \\
\text { Co II: } 64.2 \pm 9.9\end{array}$ & n.a. & & & & $\begin{array}{c}\text { RR } 0.86 \\
(0.29-2.29) \\
p=\text { n.s. }\end{array}$ & \\
\hline Byrd, 2020 [30] & $\begin{array}{l}\text { Ca: } 765 \\
\text { Co: } 1986\end{array}$ & $\begin{array}{l}\text { Ca: } M=462 \\
\text { Co: } M=846\end{array}$ & $\begin{array}{c}\text { Ca: } 58.2 \pm 9.2 \\
\text { Co: } 54.5 \pm 10.9\end{array}$ & $\begin{array}{c}\text { Ca: } \\
10.9 \pm 3.7 \mathrm{~g} / 1000 \mathrm{kcal} / \mathrm{d} \\
\mathrm{Co:} \\
11.3 \pm 3.9 \mathrm{~g} / 1000 \mathrm{kcal} / \mathrm{d}\end{array}$ & $\begin{array}{l}\text { colorectal } \\
\text { adenoma }\end{array}$ & n.a. & n.a. & $\begin{array}{c}\text { OR } 0.82 \\
(0.70-0.95) \\
p=0.01\end{array}$ & none \\
\hline $\mathrm{Fu}, 2014$ [31] & $\begin{array}{l}\text { Ca: } 1315 \\
\text { Co: } 3184\end{array}$ & $\begin{array}{l}\text { Ca: } M=913 \\
\text { Co: } M=1732\end{array}$ & $\begin{array}{l}\text { Ca: } 59.2 \\
\text { Co: } 57.5\end{array}$ & $\begin{array}{l}\text { Ca: } 17.0 \pm 1.0 \mathrm{~g} / \mathrm{d} \\
\text { Co: } 17.3 \pm 1.0 \mathrm{~g} / \mathrm{d}\end{array}$ & $\begin{array}{l}\text { colorectal } \\
\text { adenoma }\end{array}$ & Ca: 278 & $24.8 \mathrm{~g} / \mathrm{d}$ & $\begin{array}{l}\text { OR } 0.85 \\
(0.68-1.06) \\
p=0.06\end{array}$ & $\begin{array}{l}\text { Age, sex, sites, education, } \\
\text { smoking, PA, food supplement, } \\
\text { dietary calcium and folate } \\
\text { intake, and TEn }\end{array}$ \\
\hline Fuchs, 1999 [32] & $\begin{array}{l}27,530 \\
\text { Ca: } 787\end{array}$ & only F & 49 & n.a. & $\begin{array}{l}\text { colorectal } \\
\text { adenoma }\end{array}$ & Ca: 212 & $24.9 \pm 5.5 \mathrm{~g} / \mathrm{d}$ & $\begin{array}{l}\text { RR: } 0.91 \\
(0.71-1.16) \\
p=0.36\end{array}$ & $\begin{array}{c}\text { Age, smoking, BMI, PA, } \\
\text { aspirin use, family history of } \\
\text { CRC, history of colorectal } \\
\text { adenoma, red meat intake, } \\
\text { alcohol, TEn, folate, } \\
\text { methionine, calcium, vitamin } \\
\text { D intake }\end{array}$ \\
\hline Giovannucci, 1992 [33] & $\begin{array}{c}7284 \\
\text { Ca: } 170\end{array}$ & only $\mathrm{M}$ & 40-75 (range) & n.a. & $\begin{array}{l}\text { colorectal } \\
\text { adenoma }\end{array}$ & n.a. & $\geq 28.3 \mathrm{~g} / \mathrm{d}$ & $\begin{array}{l}\text { RR: } 0.36 \\
(0.22-0.60) \\
p<0.0001\end{array}$ & $\begin{array}{l}\text { Age, TEn, and family history } \\
\text { of CRC }\end{array}$ \\
\hline Haile, 1997 [34] & $\begin{array}{l}\text { Ca: } 488 \\
\text { Co: } 488\end{array}$ & $\begin{array}{l}\text { Ca: } M=325 \\
\text { Co: } M=325\end{array}$ & $\begin{array}{l}\text { Ca: } 61.9 \pm 6.7 \\
\text { Co: } 61.8 \pm 6.8\end{array}$ & $\begin{array}{l}\text { Ca: } 19.1 \mathrm{~g} / \mathrm{d} \\
\text { Co: } 20.1 \mathrm{~g} / \mathrm{d}\end{array}$ & $\begin{array}{l}\text { colorectal } \\
\text { adenoma }\end{array}$ & n.a. & $27.6 \mathrm{~g} / \mathrm{d}$ & $\begin{array}{c}\text { OR: } 0.52 \\
(0.33-0.80) \\
p<0.01\end{array}$ & $\begin{array}{l}\text { BMI, TEn, PA, smoking } \\
\text { and ethnicity }\end{array}$ \\
\hline Haslam, 2017 [35] & $\begin{array}{c}24,251 \\
\text { Ca: } 4063\end{array}$ & Ca: $\mathrm{M}=1418$ & 55-74 (range) & n.a. & $\begin{array}{l}\text { colorectal } \\
\text { adenoma }\end{array}$ & Ca: 796 & $26.8 \pm 10.8 \mathrm{~g} / \mathrm{d}$ & $\begin{array}{l}\text { OR } 0.57 \\
(0.52-0.63) \\
p<0.0001\end{array}$ & none \\
\hline
\end{tabular}


Table 2. Cont.

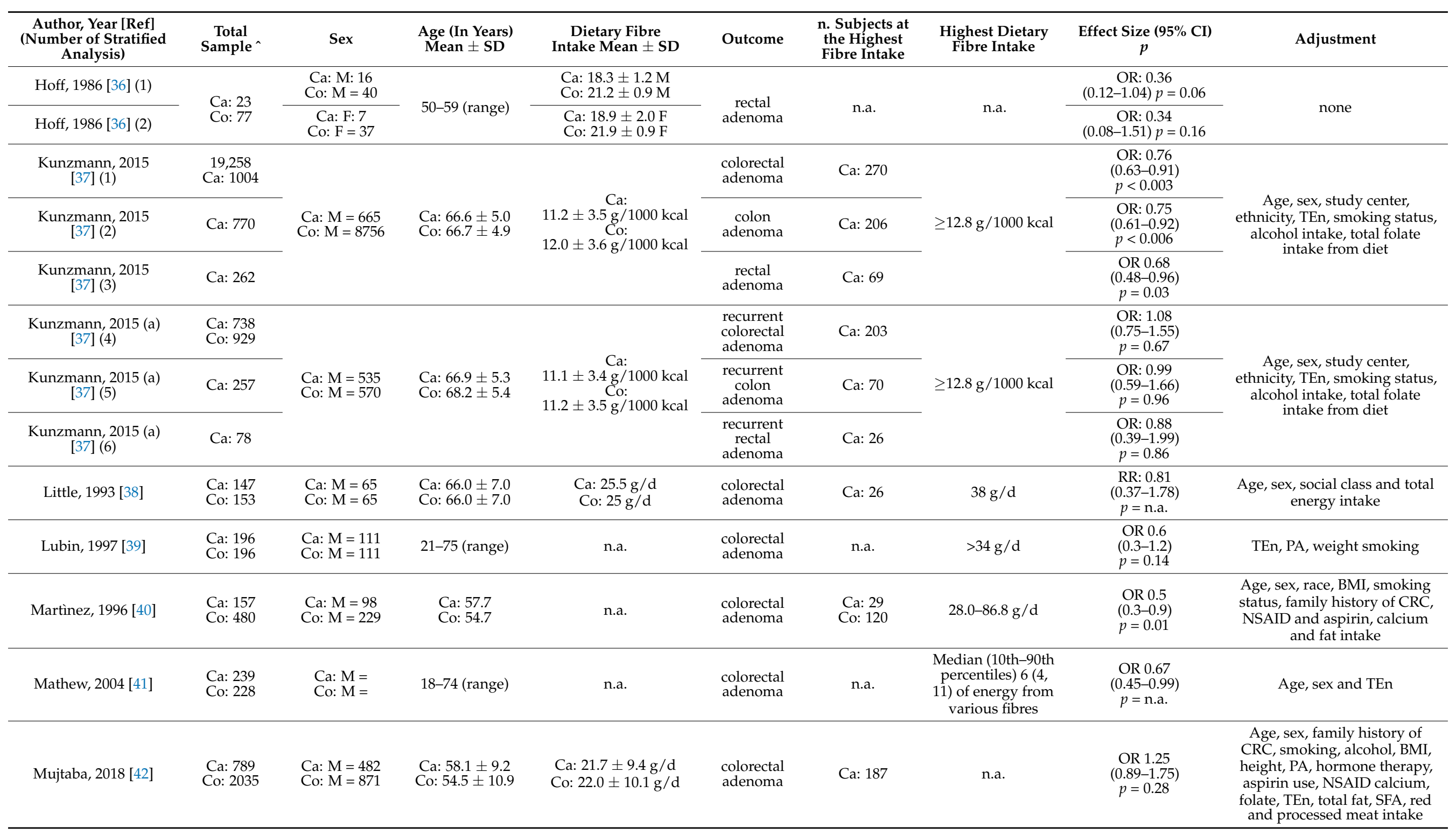


Table 2. Cont.

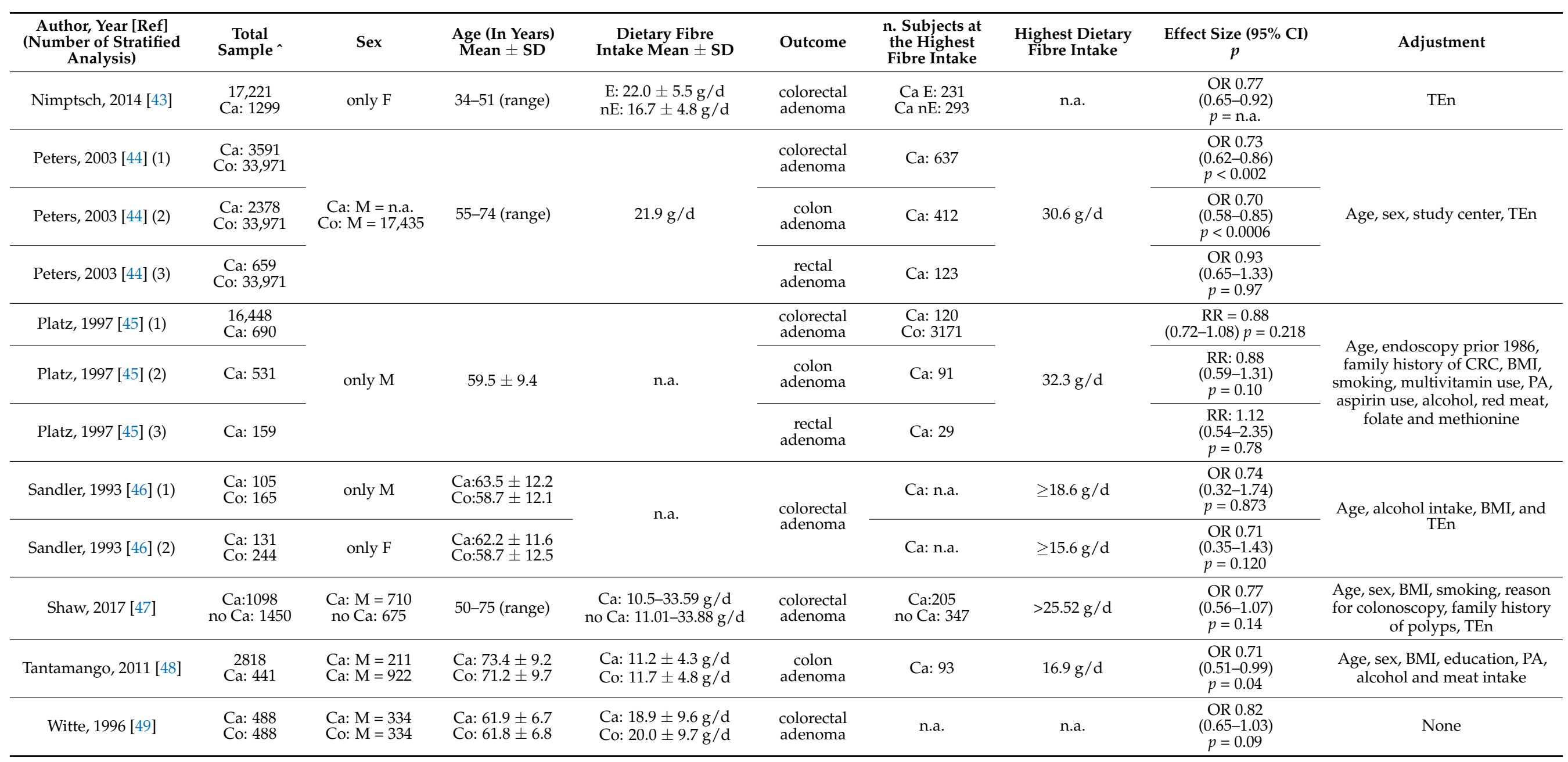

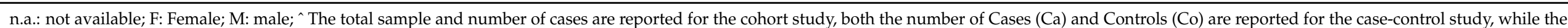

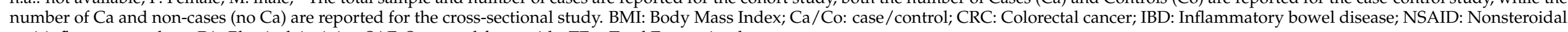
anti-inflammatory drug; PA: Physical Activity; SAF: Saturated fatty acids; TEn: Total Energy intake. 
Table 3. Results of the sensitivity and subgroup analyses.

\begin{tabular}{|c|c|c|c|c|c|c|c|c|c|c|c|}
\hline Analysis & Model & $\begin{array}{l}n . \text { Studies } \\
\text { Included }\end{array}$ & ES & $95 \%$ CI & $p$ & Sample Size & $\mathbf{I}^{2}$ & $p$ & Intercept & Tau (t) & $p$ \\
\hline \multirow{2}{*}{ Trim and Fill colorectal } & Fixed * & \multirow{2}{*}{24} & 0.71 & $0.68-0.75$ & 0.000 & \multirow{2}{*}{157,725} & \multirow{2}{*}{62.71} & \multirow{2}{*}{0.000} & \multirow{2}{*}{0.12} & \multirow{2}{*}{0.21} & \multirow{2}{*}{0.838} \\
\hline & Random ^ $^{\wedge}$ & & 0.74 & $0.67-0.82$ & 0.000 & & & & & & \\
\hline \multirow{2}{*}{$\begin{array}{l}\text { Excluding potential } \\
\text { overlapping cohort }\end{array}$} & Fixed & \multirow{2}{*}{19} & 0.79 & $0.74-0.85$ & 0.000 & \multirow{2}{*}{87,629} & \multirow{2}{*}{13.00} & \multirow{2}{*}{0.295} & \multirow{2}{*}{-0.89} & \multirow{2}{*}{-1.93} & \multirow{2}{*}{0.070} \\
\hline & Random & & 0.79 & $0.73-0.86$ & 0.000 & & & & & & \\
\hline \multirow{2}{*}{$\begin{array}{l}\text { Excluding studies with } \\
\text { estimated OR }\end{array}$} & Fixed & \multirow{2}{*}{18} & 0.76 & $0.71-0.82$ & 0.000 & \multirow{2}{*}{115,311} & \multirow{2}{*}{39.33} & \multirow{2}{*}{0.045} & \multirow{2}{*}{-0.84} & \multirow{2}{*}{-1.41} & \multirow{2}{*}{0.179} \\
\hline & Random & & 0.74 & $0.67-0.83$ & 0.000 & & & & & & \\
\hline \multirow{2}{*}{$9 y \mathrm{FU}$} & Fixed & \multirow{2}{*}{4} & 0.79 & $0.71-0.88$ & 0.000 & \multirow{2}{*}{56,453} & \multirow{2}{*}{0.00} & \multirow{2}{*}{0.595} & \multirow{2}{*}{0.15} & 007 & $0950>0$ \\
\hline & Random & & 0.79 & $0.71-0.88$ & 0.000 & & & & & 0.07 & 0.950 \\
\hline Volidatod FEO & Fixed & 15 & 0.71 & $0.68-0.75$ & 0.000 & 115102 & 7400 & & & & \\
\hline vandated ггQ & Random & 15 & 0.75 & $0.66-0.86$ & 0.000 & 115,192 & 74.88 & 0.000 & 1.16 & 1.14 & 0.276 \\
\hline Diaonosis & Fixed & 21 & 0.70 & $0.66-0.73$ & 0.000 & 120530 & 6452 & 0000 & 009 & 014 & 0891 \\
\hline Diagnosis & Random & 21 & 0.71 & $0.63-0.80$ & 0.000 & $1 \angle 0,000$ & 64.52 & 0.000 & 0.09 & 0.14 & 0.891 \\
\hline Quality score $>7$ & Fixed & 20 & 0.78 & $0.73-0.83$ & 0.000 & 125,561 & 3810 & 0.044 & -099 & -196 & 0065 \\
\hline Colon adonom & Fixed & 4 & 0.73 & $0.65-0.83$ & 0.000 & 74714 & 000 & 0774 & 120 & 120 & $0352>2>>0$ \\
\hline Coron auenoma & Random & 4 & 0.73 & $0.65-0.83$ & 0.000 & $/ 4, / 14$ & 0.00 & $0.1 / 4$ & $1 . \angle 0$ & 1.20 & 0.302 \\
\hline Postolodonomo & Fixed & 5 & 0.77 & $0.62-0.96$ & 0.019 & 69905 & 2054 & & & & \\
\hline Rectal auenoma & Random & 5 & 0.76 & $0.56-1.03$ & 0.074 & 09,900 & 30.54 & 0.218 & -0.98 & -0.86 & 0.455 \\
\hline Cohort sturdine (incidonco) & Fixed & 10 & 0.67 & $0.63-0.71$ & 0.000 & 125506 & 7520 & $0 \cap 00$ & 122 & 112 & $0207>$ \\
\hline Conort stuares (mictuence) & Random & 10 & 0.72 & $0.61-0.84$ & 0.000 & 135,506 & 75.38 & 0.000 & 1.32 & 1.12 & 0.291 \\
\hline Case-Control/Cross-sectional & Fixed & 17 & 0.78 & $0.72-0.84$ & 0.000 & 55.401 & & & & 89 & \\
\hline (prevalence) & Random & 17 & 0.76 & $0.69-0.85$ & 0.000 & 50,401 & 25.13 & 0.165 & -0.86 & -189 & 0.079 \\
\hline Women & Fixed & 6 & 0.81 & $0.70-0.92$ & 0.002 & 35152 & 000 & 0745 & & & $0240>>0$ \\
\hline Women & Random & b & 0.81 & $0.70-0.92$ & 0.002 & 30,102 & 0.00 & 0.745 & -0.50 & -1.06 & 0.349 \\
\hline Men & Fixed & 6 & 0.69 & $0.58-0.82$ & 0.000 & 24.426 & & & & & \\
\hline Men & Random & 6 & 0.46 & $0.27-0.78$ & 0.004 & $24,4 \angle 6$ & 72.73 & 0.003 & -2.24 & -2.72 & 0.053 \\
\hline
\end{tabular}



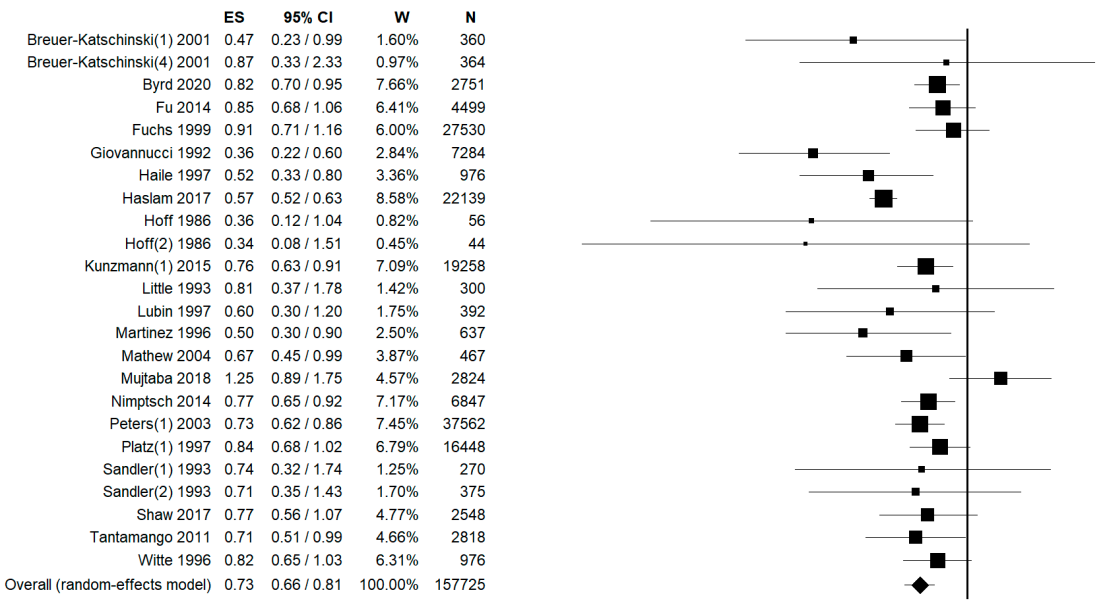

(a)

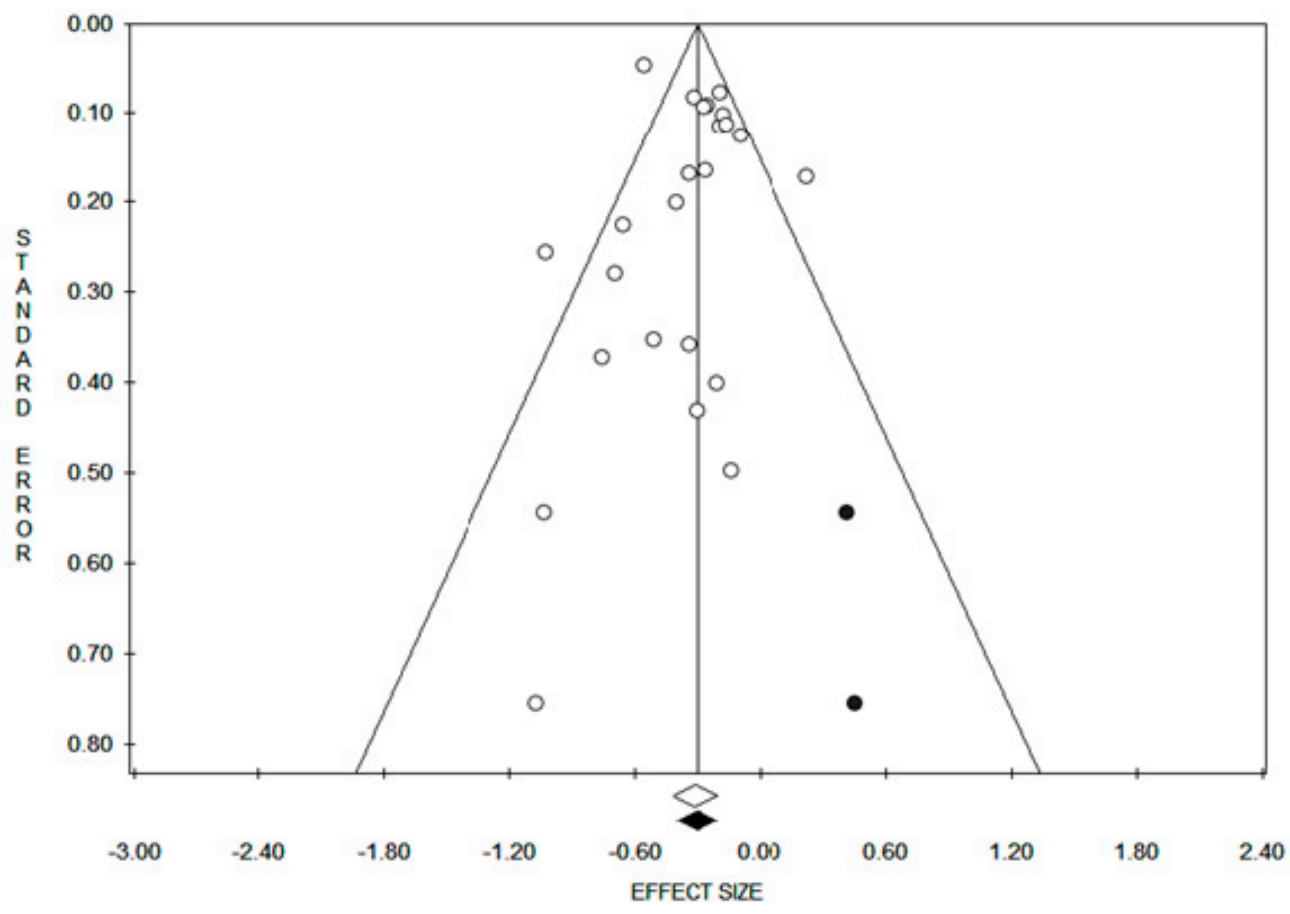

(b)

Figure 2. (a) Forest plot and (b) Funnel plot (after trim and fill method) of the meta-analysis comparing dietary fibre intake (lower vs higher intake) and risk of colorectal adenoma (random effect model). In (a) squares represent the effect size values of the individual studies. In (b) white dots represent single studies included. The black dots represent estimated studies after the trim and fill method. The white diamond represents the overall effect size of the included studies. The black diamond represents the estimated overall effect size after the trim and fill method.

No publication bias was found when considering the fixed effect model, as demonstrated by the symmetry of the Funnel plot and confirmed by Egger's linear regression Test (Intercept $0.12, t=0.21, p=0.838$ ) (Figure $2 b$ ). However, a potential publication bias was found when the random effect model was considered. In this case, we applied the trim and fill method and, after trimming 2 studies, we obtained an estimated pooled ES $=0.71$ $(95 \% \mathrm{CI}=0.68-0.75), \mathrm{p}=0.000$ in the fixed effect model and an estimated pooled $\mathrm{ES}=0.74$ $(95 \% \mathrm{CI}=0.67-0.82), \mathrm{p}=0.000$ in the random effect model (Table 3$)$. With regard to the dose-response meta-regression analysis, all studies reported dietary fibre as grams per day 
(g/d), but two studies reported dietary fibre as $\mathrm{g} / 1000 \mathrm{kcal} / \mathrm{d}[30,37]$. In view of this, we assumed a standard daily energy intake of $2000 \mathrm{kcal}$ and, for this reason we multiplied the reported intake by two. Figure 3 shows the meta-regression plot of the log OR (colorectal adenoma) on the dose of dietary fibre intake, using the fixed effect (Figure 3a) and the random effect models (Figure 3b). The size of the circles (identifying the included studies) denotes the study's weight which is represented by the inverse of the within-study variance in the fixed model, and the total variance for each study in the random effect model. The line shows the predicted values where, in the fixed effect model, the intake of dietary fibre showed negative weak-border line significant correlation with colorectal adenoma $(\mathrm{Y}=-0.06, \mathrm{z}=-0.02, \mathrm{p}=0.056)$, whilst in the random effect model, the linear correlation was not significant $(Y=-0.01, z=-0.01, p=0.095)$.

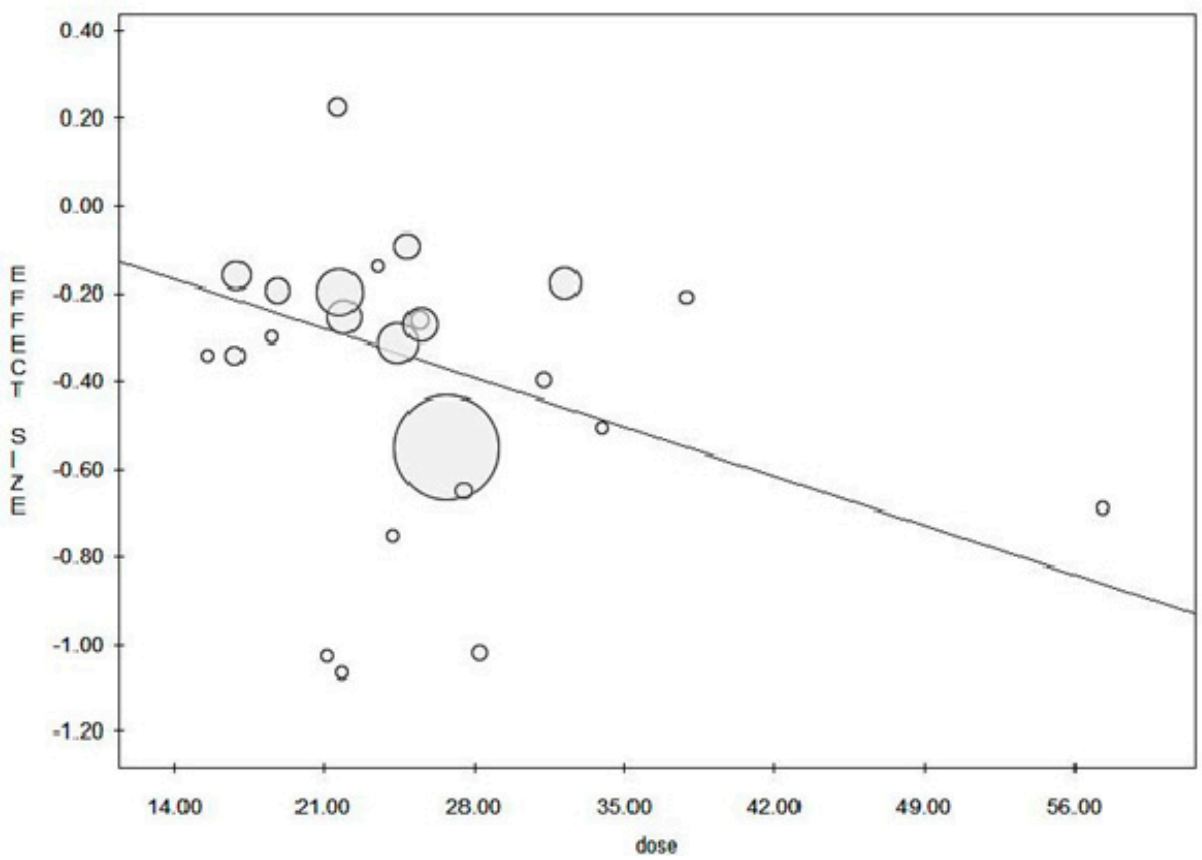

(a)

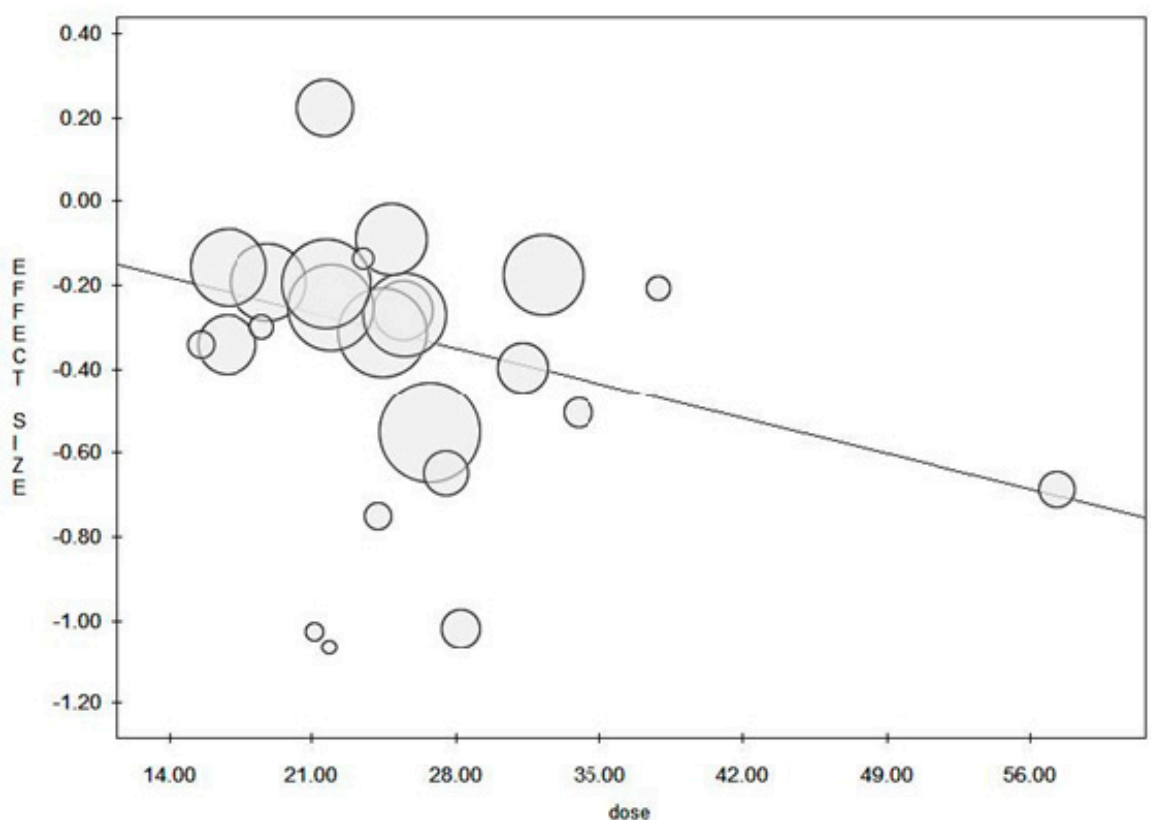

(b)

Figure 3. Meta-regression plot for (a) fixed effect model and (b) random effect model. 


\subsection{Sub-Group and Sensitivity Analysis}

A series of sensitivity analyses were conducted in order to confirm the robustness of our results. Firstly, we removed those studies that used the same cohort in order to reduce ES overestimation due to the potential overlapping effect. In particular, four studies were excluded from the analysis $[30,33,35,44]$, thus obtaining similar results compared to the main analysis but with no statistical heterogeneity $\left(\mathrm{I}^{2}=13.00 \%, p=0.295\right)$ (Table 3 ). Potential publication bias was found, as confirmed by Egger's Linear Regression Test (Intercept $-0.89, \mathrm{t}=-1.93, p=0.070$ ). Also, in this case, a trim and fill method was applied by trimming six studies on the right and the results did not change [fixed effect estimated $\mathrm{ES}=0.79(95 \% \mathrm{CI}=0.74-0.85), p=0.000$; the random effect model estimated $\mathrm{ES}=0.82$ $(95 \% \mathrm{CI}=0.77-0.91), p=0.000)]$.

Secondly, we removed studies for which OR was computed based on data reported in the original studies $[30,35,36,45,49]$, obtaining similar results with no heterogeneity and an absence of potential publication bias as confirmed by Egger's Linear Regression Test (Table 3). Thirdly, only those studies with an FU equal to or higher than 9 years were included; the results based on 53,827 participants did not materially change and no statistical heterogeneity $\left(\mathrm{I}^{2}=0.00 \%, p=0.018\right)$ was found (Table 3$)$. No publication bias was found as confirmed by Egger's Linear Regression Test (Intercept 0.15, $t=0.07, p=0.950$ ). Fourthly, results were not affected by limiting the analysis to only include studies that used validated tools to assess dietary fibre intake; however, a high statistical heterogeneity was found (Table 3) but with no publication bias.

Stronger results were obtained when limiting the pooling of studies by only using colonoscopy and histopathological confirmation for the diagnosis of adenoma and for studies with a quality score higher than 7 [low statistical heterogeneity $\left(\mathrm{I}^{2}=38.10 \%\right.$, $p=0.007$ ) but with potential publication bias (Egger's Linear Regression Test Intercept $-0.99, \mathrm{t}=-1.96, p=0.065)$; however, the trim and fill method, by trimming 6 studies on the right, did not change results: in the fixed effect model, the pooled ES was 0.81 $(95 \% \mathrm{CI}=0.76-0.86), p=0.000$; in the random effect model ES was $0.82(95 \% \mathrm{CI}=0.73-0.91)$ $p=0.000]$. In order to corroborate the results obtained, sub-group analyses were additionally performed, based on the adenoma site (colon and rectal separately), study design (case-control/cross-sectional and cohort studies) and sex. Results also remained similar in these cases. We detected a potential publication bias in the subgroup analyses by study design and sex when only including case control/cross-sectional studies and men, respectively. However, ES estimated after the trim and fill method did not change. Data are shown in Table 3. Results should be interpreted with caution for adenoma site and sex since a few studies for each analysis were pooled and also due to the low number of stratified analyses conducted in the original studies retrieved.

\subsection{Cumulative Analysis}

The cumulative analysis in Figure 4a reveals that early studies, even if suggesting an inverse association between fibre intake and risk of colorectal adenoma, had a wide $95 \%$ CI which decreased in the late 1990s and relatively stabilized during the first decade of 2000. However, a really narrow $95 \% \mathrm{CI}$ and a consistent ES was obtained during the second decade of 2000. With reference to the cumulative analysis by sample size (Figure $4 b$ ), studies with a sample size smaller than 1000 subjects, even if establishing an inverse statistically-significant association, had a wide $95 \%$ CI. As expected, a larger sample size contributed to stabilizing the results both by reducing the $95 \% \mathrm{CI}$ and by corroborating the ES value. Considering the cumulative analysis by dietary fibre dose, ES was higher when studies examined a dietary dose lower than $26 \mathrm{~g} / \mathrm{d}$. Conversely, ES was lower and stable and with a narrow $95 \% \mathrm{CI}$, when higher dietary fibre intake was considered (Figure 4c). 


$\begin{array}{rlcr} & \text { ES } & 95 \% \text { Cl } & \text { N } \\ \text { Hoff 1986 } & 0.36 & 0.12 / 1.04 & 56 \\ \text { Hoff(2) 1986 } & 0.35 & 0.15 / 0.84 & 100 \\ \text { Giovannucci 1992 } & 0.36 & 0.23 / 0.55 & 7384 \\ \text { Little 1993 } & 0.43 & 0.30 / 0.63 & 7684 \\ \text { Sandler(1) 1993 } & 0.47 & 0.34 / 0.67 & 7954 \\ \text { Sandler(2) 1993 } & 0.51 & 0.38 / 0.70 & 8329 \\ \text { Martinez 1996 } & 0.51 & 0.39 / 0.67 & 8968 \\ \text { Witte 1996 } & 0.67 & 0.57 / 0.80 & 9942 \\ \text { Haile 1997 } & 0.65 & 0.55 / 0.77 & 10918 \\ \text { Lubin 1997 } & 0.65 & 0.55 / 0.76 & 11310 \\ \text { Platz(1) 1997 } & 0.71 & 0.63 / 0.81 & 27758 \\ \text { Fuchs 1999 } & 0.75 & 0.67 / 0.84 & 55288 \\ \text { Breuer-Katschinski(1) 2001 } & 0.74 & 0.67 / 0.83 & 55848 \\ \text { Breuer-Katschinski(4) 2001 } & 0.74 & 0.67 / 0.83 & 58012 \\ \text { Peters(1) 2003 } & 0.74 & 0.68 / 0.81 & 93574 \\ \text { Mathew 2004 } & 0.74 & 0.67 / 0.80 & 94041 \\ \text { Tantamango 2011 } & 0.73 & 0.67 / 0.80 & 98859 \\ \text { Fu 2014 } & 0.75 & 0.69 / 0.81 & 101358 \\ \text { Nimptsch 2014 } & 0.75 & 0.70 / 0.81 & 108205 \\ \text { Kunzmann(1) 2015 } & 0.75 & 0.70 / 0.81 & 127463 \\ \text { Haslam 2017 } 2020 & 0.69 & 0.65 / 0.72 & 149602 \\ \text { Shaw 2017 } & 0.69 & 0.65 / 0.73 & 152150 \\ \text { Mujtaba 2018 } & 0.70 & 0.68 / 0.74 & 154974 \\ & 0.71 & 0.68 / 0.75 & 157725\end{array}$

(a)

Figure 4. Cont. 


$\begin{array}{rlcr} & \text { ES } & 95 \% \text { Cl } & \text { N } \\ \text { Hoff(2) 1986 } & 0.34 & 0.08 / 1.51 & 44 \\ \text { Hoff 1986 } & 0.35 & 0.15 / 0.84 & 100 \\ \text { Sandler(1) 1993 } & 0.51 & 0.28 / 0.94 & 370 \\ \text { Little 1993 } & 0.61 & 0.38 / 0.98 & 670 \\ \text { Breuer-Katschinski(1) 2001 } & 0.56 & 0.38 / 0.84 & 1030 \\ \text { Breuer-Katschinski(4) 2001 } & 0.60 & 0.41 / 0.87 & 1394 \\ \text { Sandler(2) 1993 } & 0.62 & 0.45 / 0.86 & 1769 \\ \text { Lubin 1997 } & 0.62 & 0.46 / 0.83 & 2161 \\ \text { Mathew 2004 } & 0.64 & 0.50 / 0.81 & 2628 \\ \text { Martinez 1998 } & 0.61 & 0.49 / 0.76 & 3265 \\ \text { Haile 1997 } & 0.59 & 0.49 / 0.72 & 4241 \\ \text { Witte 1998 } & 0.68 & 0.59 / 0.79 & 5217 \\ \text { Shaw 2017 } & 0.70 & 0.61 / 0.80 & 7765 \\ \text { Byrd 2020 } & 0.75 & 0.68 / 0.83 & 10516 \\ \text { Tantamango 2011 } & 0.74 & 0.68 / 0.82 & 13334 \\ \text { Mujtaba 2018 } & 0.77 & 0.71 / 0.85 & 16158 \\ \text { Fu 2014 } & 0.79 & 0.72 / 0.86 & 20657 \\ \text { Nimptsch 2014 } & 0.78 & 0.72 / 0.85 & 27504 \\ \text { Giovannucci 1992 } & 0.77 & 0.71 / 0.83 & 34788 \\ \text { Platz(1) 1997 } & 0.78 & 0.72 / 0.83 & 51236 \\ \text { Kunzmann(1) 2015 } & 0.77 & 0.72 / 0.83 & 70494 \\ \text { Haslam 2017 } & 0.70 & 0.68 / 0.74 & 92633 \\ \text { Fuchs 1999 } & 0.71 & 0.67 / 0.75 & 120163 \\ 2003 & 0.71 & 0.68 / 0.75 & 157725\end{array}$

(b)

Figure 4. Cont. 
Sandler(2) 1993

Tantamango 2011

Fu 2014

Sandler(1) 1993

Witte 1996

Hoff 1986

Mujtaba 2018

Byrd 2020

Hoff(2) 1986

Nimptsch 2014

Breuer-Katschinski(4) 2001

Breuer-Katschinski(1) 2001

Peters(1) 2003

Fuchs 1999

Shaw 2017

Kunzmann(1) 2015

Haslam 2017

Haile 1997

Giovannucci 1992

Mathew 2004

Platz(1) 1997

Lubin 1997

Little 1993

Martinez 1996

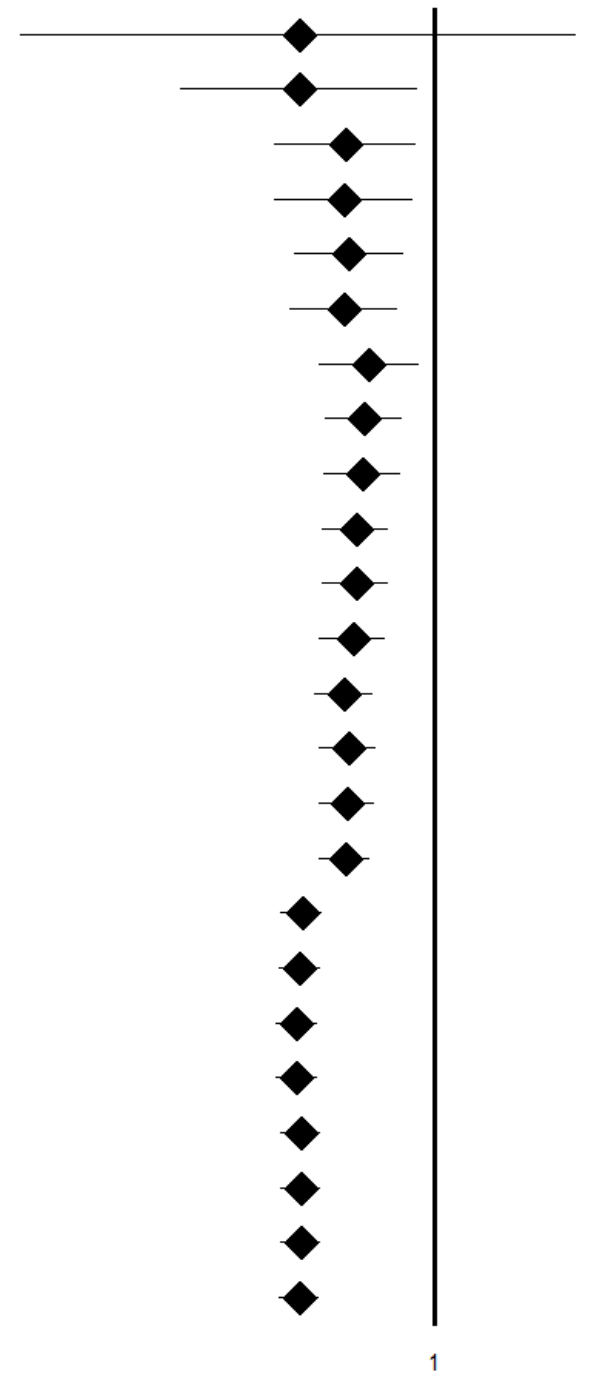

(c)

Figure 4. Forest plot of the cumulative analysis by (a) year of publication (from the first to the most recent published study), (b) by sample size (from the smallest to the largest), (c) dietary fibre dose (from the lowest to the highest intake) between dietary fibre intake and risk of colorectal adenoma. Diamonds represent the effect size estimated using the cumulative analysis calculated adding one study at a time.

\section{Discussion}

This is an extensive systematic review and meta-analysis of observational studies conducted by searching three different databases (PubMed/Medline, Scopus and EMBASE) and assessing the association between dietary fibre intake and the risk of colorectal adenoma. Our meta-analysis of 21 studies in total found an approximate 30\% risk reduction of adenoma associated with a higher intake of dietary fibre. This result was confirmed in both the fixed and random effect models. Moreover, meta-regression analysis was performed since the original studies reported dietary fibre intake homogeneously. Meta-regression analysis predicts the changes of the outcome (colorectal adenoma) for a unit increase in dietary fibre intake. Our results showed a border-line significant negative linear correlation between the amount of dietary fibre intake and colorectal adenoma (the higher the intake of dietary fibre, the lower the risk of colorectal adenoma). This observation is in line with the cumulative analysis by dietary fibre dose, according to which the lowest risk of adenoma was associated with a dietary fibre intake equal to or higher than $26 \mathrm{~g} / \mathrm{d}$. However, it 
should be noted that the ES from the pooling of the 21 studies was associated with moderate heterogeneity and a potential publication bias. For these reasons, we performed several sensitivity analyses and the trim and fill method was applied. With regard to the potential publication bias, the trim and fill methods trimmed two possible studies on the right, however, the result did not change. Looking at the subgroup analysis by sex, a higher protective effect for men was found when compared to women, yet there was higher heterogeneity in men than in women. Furthermore, the highest strength of the association between dietary fibre intake and colorectal adenoma was found when focusing on cohort studies wherein higher heterogeneity was found when compared to case-control/cross-sectional studies. This is probably because a different duration of FU was considered among the original pooled cohort studies, and this hypothesis can be confirmed when considering a sensitivity analysis limited to 9 years (or more) of FU, where heterogeneity dramatically dropped. Moreover, the natural history of colorectal adenoma should be considered when interpreting these results. On the one hand, colorectal adenoma is characterized by a long latency period, which might not be better appraised in short cohort studies, nor in long cohort studies, where maintaining an FU might be difficult while increasing the risk of selection bias. On the other hand, case-control studies are more prone to potential recall bias. However, the high number of retrieved studies and consequently, the large sample size, might mitigate the risk. An important aspect that should be considered is that the vast majority of included studies were conducted in the USA, a population that largely did not meet healthy dietary guidelines, and where the eating pattern of approximately three-fourths of the population is low in vegetables, fruits, dairy, and oils and rich in refined grain, proteins, saturated fats, sodium and total calories [50]. This dietary pattern is frequently associated with several preventable, diet-related chronic diseases, including cancers. Based on this, we can speculate that the healthy beneficial effects of dietary fibre intake might be higher with respect to what we found in this meta-analysis if assessed in a population with a more Mediterranean—or, generally speaking, healthier-dietary pattern. It should be considered that the most relevant source of dietary fibre derives from vegetables, legumes and fruits which have been shown to prevent colorectal cancer [51] and which are heterogeneous in respect to their composition; therefore, it can be assumed that they have various anticarcinogenic properties. They are rich in bioactive compounds such as vitamins, anti-oxidants and polyphenols. Previous in vitro studies showed that some of these polyphenols, such as sulforaphane and epigallocatechin, are able to reprogram gene expression through epigenetic modification, thus reverting cancer progression $[52,53]$. Moreover, it should be considered that almost all the included studies used FFQs to assess dietary fibre intake, and even if they were frequently validated, it is difficult to precisely estimate the intake, often resulting in underestimation. Furthermore, our results suggest that there might be differences in the responses to fibre by sex.

\subsection{Potential Biological Mechanisms}

Since colorectal adenomas are considered to be potential precancerous lesions, they are likely to share a common etiopathogenesis with colorectal cancer.

According to the European Food Safety Authority (EFSA) dietary fibre is "non-digestible carbohydrates plus lignin, including non-starch polysaccharides, fructo-oligosaccharides, galactooligosaccharides, other resistant oligosaccharides and resistant" [54]. Major food sources for dietary fibre are cereals/grains, vegetables, fruits and legumes. Based on its components, previous studies suggested to differentiate dietary fibre into "soluble" and "insoluble". Such distinction was used to differentiate between viscous, soluble types of fibre (e.g., pectins) and insoluble components such as cellulose. Even if the distinction was mainly proposed to identify different patterns of beneficial effects, it should be noticed that both soluble and insoluble components have different and synergic advantages. The insoluble fibre is mainly responsible for the increase in stool bulk, important for reducing transit time and diluting carcinogens in the lumen by means of both reducing exposure to carcinogens and lowering secondary bile acid production [5]. The soluble fibre, instead, 
seems to be implicated in the wellbeing of microbiota through fibre fermentation which, in turn, is able to promote the production of short-chain fatty acids (SCFA) and which, by lowering the colonic $\mathrm{pH}$, might inhibit pathogenic microorganisms and increase the absorption of some nutrients [55]. In addition, experimental studies have shown that butyrate, SCFA, has anti-proliferative effects, promotes colon motility and induces apoptosis [56]. The consequent reduction in cholesterol, and insulin resistance, seem to inherently reduce the risk of colorectal cancer. Furthermore, dietary fibre intake seems to also promote the eubiosis of the gut microbiota ecosystem [57]. On the contrary, dysbiosis (increased number of harmful bacteria in the gut) seems to be associated with an increased release of enterotoxins that alter the immune system, inducing the production of pro-inflammatory cytokines responsible for the disease status [58], including colorectal cancer [59].

Considering the beneficial effects of both dietary fibre components, and based on the suggestion provided by Food and Agriculture Organization (FAO) and World Health Organization (WHO) stating that the above mentioned distinction should be overcome as solubility does not always predict physiological effects [60], we did not perform a separate analysis among soluble and insoluble fibre. Indeed, the potential role of fibre in preventing colorectal adenoma and then cancer could be attributed to all of the mentioned mechanisms, mainly on account of the dietary fibre heterogeneity in chemical composition, physicochemical properties, and solubility. On the other hand, selectively focusing on one of the two components and consequently on a particular food source might lead to a reduction in diet variety. In light of this, international healthy dietary guidelines consider dietary fibre as a single entity [61] and recommend satisfying the daily dietary fibre intake of at least $30 \mathrm{~g}$, derived from a varied and balanced diet, rich in plant-based foods, such as wholegrains, legumes, non-starchy vegetables and fruit [62], as the Mediterranean diet advocates [63]. Nevertheless, in our society, the increased rate of colorectal adenoma (and cancer) can be attributed, among the others, to unhealthy lifestyles, including the so-called Western diet. This dietary pattern is characterized on one hand by a high intake of refined grains, sugars, salt, saturated and trans-fatty acids mainly due to a high consumption of ultra-processed food $[64,65]$, and on the other hand by a low intake of dietary fibre. In this respect, previous studies showed that a low intake of fibre along with a high consumption of typical western diet food increase the risk of dysbiosis, which in turn can be responsible for a lower production in SCFA $[66,67]$.

\subsection{Strengths and Limitations}

This meta-analysis is affected by some limitations directly stemming from the included studies. First of all, dietary fibre intake was self-reported, most of the time using FFQ which, even if validated, cannot prevent recall bias or social desirability bias, thus both resulting in mis-reporting. Secondly, information on cooking methods were not assessed and reported in the original studies, and recent evidence showed a modification in the fibre structure and biological effect based on the processing method used [68,69]. Thirdly, in the main analysis, the funnel plot confirmed by the Egger's test showed a potential publication bias. However, when applying the trim and fill methods the estimated ES did not change when compared to the observed ES. Another possible limitation is the language used (only articles published in English), which can lead to a bias in the selection of studies. Since English is the commonly-used scientific language, we believe that this did not affect our results as the most relevant and high-quality articles are published in English. Despite the above-mentioned limitations, our review with meta-analysis has important strengths. Worth highlighting is that this is a systematic and extensive review of available evidence, conducted according to the main guidelines. Furthermore, we conducted several sensitivity and sub-group analyses that make our results stronger and consistent. All the sub-group analyses performed offer a deep understanding of the association, useful for public health experts, dietitians and clinicians-the former involved in issuing health-related policies and campaigns and the latter in educating and assisting patients. Moreover, we were able to perform a meta-regression analysis as well as a cumulative analysis, confirming the 
association between a higher intake of dietary fibre and a lower risk of colorectal adenoma. Lastly, in our meta-analysis we pooled ES with a higher level of adjustment than was reported in each original study.

\section{Conclusions}

To conclude, the results of our systematic review with meta-analysis show a statistically significant and robust association between a higher intake of dietary fibre and a lower risk of colorectal adenoma, both considering a prevalent and incident risk of adenoma (as confirmed by the subgroup analysis by study design). These results are extremely important because we were able to estimate the reduction in the risk of the development of a preneoplastic lesion as a result of a higher dietary fibre intake, which can be considered as a valid healthy dietary recommendation. Given the relatively simple implementation of this dietary behaviour, our findings confirm the importance of reinforcing this knowledge and awareness among both health care professionals (including, but not limited to those involved in nutritional educational programmes) and the general population.

Supplementary Materials: The following are available online at https:/ /www.mdpi.com/article/10 .3390/ijerph18084168/s1, Table S1: Full search strategy; Table S2: Studies excluded with reasons after full-text assessment; Table S3: Quality assessment of the included studies.

Author Contributions: V.G. and D.N., contributed to the conception and design of the study and search. D.N. and C.F., extracted meta-data from the included manuscripts and performed the quality assessment. V.G. performed the analyses. V.G. and D.N. drafted the manuscript and performed the analysis. D.N. contributed to the assembly of the paper and data interpretation. V.G., T.S., M.N. and S.R. helped in the literature review and provided a critical revision of drafts. All authors have read and agreed to the published version of the manuscript.

Funding: This research received no external funding.

Institutional Review Board Statement: Not applicable.

Informed Consent Statement: Not applicable.

Conflicts of Interest: The authors declare no conflict of interest.

\section{References}

1. International Agency for Research on Cancer. World Cancer Report: Cancer Research for Cancer Prevention; Wild, C.P., Weiderpass, E., Stewart, B.W., Eds.; International Agency for Research on Cancer (IARC): Lyon, France, 2020.

2. Schreuders, E.H.; Ruco, A.; Rabeneck, L.; Schoen, R.E.; Sung, J.J.; Young, G.P.; Kuipers, E.J. Colorectal cancer screening: A global overview of existing programmes. Gut 2015, 64, 1637-1649. [CrossRef]

3. Centres for Diseases Control and Prevention. Colorectal Cancer Screening Tests. Available online: https://www.cdc.gov/cancer/ colorectal/basic_info/screening/tests.htm (accessed on 20 February 2021).

4. Fleming, M.; Ravula, S.; Tatishchev, S.F.; Wang, H.L. Colorectal carcinoma: Pathologic aspects. J. Gastrointest Oncol. 2012, 3, 153-173. [CrossRef]

5. World Cancer Research Fund. Diet, Nutrition, Physical Activity and Colorectal Cancer. Available online: https://www.wcrf.org/ sites / default/ files /Colorectal-cancer-report.pdf (accessed on 13 March 2021).

6. Gianfredi, V.; Salvatori, T.; Villarini, M.; Moretti, M.; Nucci, D.; Realdon, S. Is dietary fibre truly protective against colon cancer? A systematic review and meta-analysis. Int. J. Food Sci. Nutr. 2018, 69, 904-915. [CrossRef]

7. Gianfredi, V.; Nucci, D.; Salvatori, T.; Dallagiacoma, G.; Fatigoni, C.; Moretti, M.; Realdon, S. Rectal Cancer: 20\% Risk Reduction Thanks to Dietary Fibre Intake. Systematic Review and Meta-Analysis. Nutrients 2019, 11, 1579. [CrossRef]

8. Higgins, J.P.; Altman, D.G.; Gotzsche, P.C.; Juni, P.; Moher, D.; Oxman, A.D.; Savovic, J.; Schulz, K.F.; Weeks, L.; Sterne, J.A.; et al. The Cochrane Collaboration's tool for assessing risk of bias in randomised trials. BMJ 2011, 343, d5928. [CrossRef]

9. Stroup, D.F.; Berlin, J.A.; Morton, S.C.; Olkin, I.; Williamson, G.D.; Rennie, D.; Moher, D.; Becker, B.J.; Sipe, T.A.; Thacker, S.B. Meta-analysis of observational studies in epidemiology: A proposal for reporting. Meta-analysis Of Observational Studies in Epidemiology (MOOSE) group. JAMA 2000, 283, 2008-2012. [CrossRef] [PubMed]

10. Moher, D.; Liberati, A.; Tetzlaff, J.; Altman, D.G.; Group, P. Preferred reporting items for systematic reviews and meta-analyses: The PRISMA statement. PLoS Med. 2009, 6, e1000097. [CrossRef] [PubMed]

11. Liberati, A.; Altman, D.G.; Tetzlaff, J.; Mulrow, C.; Gotzsche, P.C.; Ioannidis, J.P.; Clarke, M.; Devereaux, P.J.; Kleijnen, J.; Moher, D. The PRISMA statement for reporting systematic reviews and meta-analyses of studies that evaluate health care interventions: Explanation and elaboration. Ann. Intern. Med. 2009, 151, W65-W94. [CrossRef] [PubMed] 
12. Higgins, J.P.T.; Green, S. Cochrane Handbook for Systematic Reviews of Interventions. Version 5.1.0.; The Cochrane Collaboration: London, UK, 2013.

13. Brown, P.; Brunnhuber, K.; Chalkidou, K.; Chalmers, I.; Clarke, M.; Fenton, M.; Forbes, C.; Glanville, J.; Hicks, N.J.; Moody, J.; et al. How to formulate research recommendations. BMJ Clin. Res. Ed. 2006, 333, 804-806. [CrossRef] [PubMed]

14. Gianfredi, V.; Salvatori, T.; Nucci, D.; Villarini, M.; Moretti, M. Can chocolate consumption reduce cardio-cerebrovascular risk? A systematic review and meta-analysis. Nutrition 2018, 46, 103-114. [CrossRef] [PubMed]

15. Gianfredi, V.; Bragazzi, N.L.; Nucci, D.; Villarini, M.; Moretti, M. Cardiovascular diseases and hard drinking waters: Implications from a systematic review with meta-analysis of case-control studies. J. Water Health 2017, 15, 31-40. [CrossRef]

16. Wells, G.A.; Shea, B.; O'Connell, D.; Paterson, J.; Welch, V.; Losos, M.; Tugwell, P. The Newcastle-Ottawa Scale (NOS) for Assessing the Quality of Nonrandomised Studies in Meta-Analyses. Available online: http://www.ohri.ca/programs/clinical_ epidemiology / oxford.asp (accessed on 15 November 2020).

17. Herzog, R.; Alvarez-Pasquin, M.J.; Diaz, C.; Del Barrio, J.L.; Estrada, J.M.; Gil, A. Are healthcare workers' intentions to vaccinate related to their knowledge, beliefs and attitudes? A systematic review. BMC Public Health 2013, 13, 154. [CrossRef]

18. Egger, M.; Davey Smith, G.; Schneider, M.; Minder, C. Bias in meta-analysis detected by a simple, graphical test. BMJ Clin. Res. Ed. 1997, 315, 629-634. [CrossRef] [PubMed]

19. Duval, S.; Tweedie, R. A nonparametric "Trim and Fill" method of accounting for Publication Bias in Meta-Analysis. J. Am. Stat. Assoc. 2000, 95, 89-98. [CrossRef]

20. Leimu, R.; Koricheva, J. Cumulative meta-analysis: A new tool for detection of temporal trends and publication bias in ecology. Proc. Biol. Sci. 2004, 271, 1961-1966. [CrossRef] [PubMed]

21. Austin, G.L.; Adair, L.S.; Galanko, J.A.; Martin, C.F.; Satia, J.A.; Sandler, R.S. A diet high in fruits and low in meats reduces the risk of colorectal adenomas. J. Nutr. 2007, 137, 999-1004. [CrossRef] [PubMed]

22. Benito, E.; Cabeza, E.; Moreno, V.; Obrador, A.; Bosch, F.X. Diet and colorectal adenomas: A case-control study in Majorca. Int. J. Cancer 1993, 55, 213-219. [CrossRef] [PubMed]

23. Haslam, A.; Wagner Robb, S.; Hébert, J.R.; Huang, H.; Ebell, M.H. Association between dietary pattern scores and the prevalence of colorectal adenoma considering population subgroups. Nutr. Diet. J. Dietit. Assoc. Aust. 2018, 75, 167-175. [CrossRef]

24. Kopp, T.I.; Vogel, U.; Tjonneland, A.; Andersen, V. Meat and fiber intake and interaction with pattern recognition receptors (TLR1, TLR2, TLR4, and TLR10) in relation to colorectal cancer in a Danish prospective, case-cohort study. Am. J. Clin. Nutr. 2018, 107, 465-479. [CrossRef]

25. Kune, G.A.; Kune, S.; Read, A.; MacGowan, K.; Penfold, C.; Watson, L.F. Colorectal polyps, diet, alcohol, and family history of colorectal cancer: A case-control study. Nutr. Cancer 1991, 16, 25-30. [CrossRef]

26. Macquart-Moulin, G.; Riboli, E.; Cornee, J.; Kaaks, R.; Berthezene, P. Colorectal polyps and diet: A case-control study in Marseilles. Int. J. Cancer 1987, 40, 179-188. [CrossRef]

27. Neugut, A.I.; Garbowski, G.C.; Lee, W.C.; Murray, T.; Nieves, J.W.; Forde, K.A.; Treat, M.R.; Waye, J.D.; Fenoglio-Preiser, C. Dietary risk factors for the incidence and recurrence of colorectal adenomatous polyps. A case-control study. Ann. Intern. Med. 1993, 118, 91-95. [CrossRef] [PubMed]

28. Zheng, X.; Nguyen, L.H.; Liu, P.H.; Wu, K.; Smith-Warner, S.; Willett, W.; Chan, A.T.; Giovannucci, E.; Cao, Y. Comprehensive assessment of diet quality and risk of early-onset colorectal adenoma. Gastroenterology 2019, 156, S208. [CrossRef]

29. Breuer-Katschinski, B.; Nemes, K.; Marr, A.; Rump, B.; Leiendecker, B.; Breuer, N.; Goebell, H.; Colorectal Adenoma Study, G. Colorectal adenomas and diet: A case-control study. Colorectal Adenoma Study Group. Dig. Dis. Sci. 2001, 46, 86-95. [CrossRef] [PubMed]

30. Byrd, D.A.; Judd, S.; Flanders, W.D.; Hartman, T.J.; Fedirko, V.; Bostick, R.M. Associations of Novel Dietary and Lifestyle Inflammation Scores with Incident, Sporadic Colorectal Adenoma. Cancer Epidemiol. Biomarkers Prev. 2020, 29, $2300-2308$. [CrossRef] [PubMed]

31. Fu, Z.; Shrubsole, M.J.; Smalley, W.E.; Ness, R.M.; Zheng, W. Associations between dietary fiber and colorectal polyp risk differ by polyp type and smoking status. J. Nutr. 2014, 144, 592-598. [CrossRef]

32. Fuchs, C.S.; Giovannucci, E.L.; Colditz, G.A.; Hunter, D.J.; Stampfer, M.J.; Rosner, B.; Speizer, F.E.; Willett, W.C. Dietary fiber and the risk of colorectal cancer and adenoma in women. N. Engl. J. Med. 1999, 340, 169-176. [CrossRef]

33. Giovannucci, E.; Stampfer, M.J.; Colditz, G.; Rimm, E.B.; Willett, W.C. Relationship of diet to risk of colorectal adenoma in men. J. Natl. Cancer Inst. 1992, 84, 91-98. [CrossRef]

34. Haile, R.W.; Witte, J.S.; Longnecker, M.P.; Probst-Hensch, N.; Chen, M.J.; Harper, J.; Frankl, H.D.; Lee, E.R. A sigmoidoscopy-based case-control study of polyps: Macronutrients, fiber and meat consumption. Int. J. Cancer 1997, 73, 497-502. [CrossRef]

35. Haslam, A.; Wagner Robb, S.; Hebert, J.R.; Huang, H.; Wirth, M.D.; Shivappa, N.; Ebell, M.H. The association between Dietary Inflammatory Index scores and the prevalence of colorectal adenoma. Public Health Nutr. 2017, 20, 1609-1616. [CrossRef]

36. Hoff, G.; Moen, I.E.; Trygg, K.; Frolich, W.; Sauar, J.; Vatn, M.; Gjone, E.; Larsen, S. Epidemiology of polyps in the rectum and sigmoid colon. Evaluation of nutritional factors. Scand. J. Gastroenterol. 1986, 21, 199-204. [CrossRef]

37. Kunzmann, A.T.; Coleman, H.G.; Huang, W.Y.; Kitahara, C.M.; Cantwell, M.M.; Berndt, S.I. Dietary fiber intake and risk of colorectal cancer and incident and recurrent adenoma in the Prostate, Lung, Colorectal, and Ovarian Cancer Screening Trial. Am. J. Clin. Nutr. 2015, 102, 881-890. [CrossRef] 
38. Little, J.; Logan, R.F.; Hawtin, P.G.; Hardcastle, J.D.; Turner, I.D. Colorectal adenomas and diet: A case-control study of subjects participating in the Nottingham faecal occult blood screening programme. Br. J. Cancer 1993, 67, 177-184. [CrossRef]

39. Lubin, F.; Rozen, P.; Arieli, B.; Farbstein, M.; Knaani, Y.; Bat, L.; Farbstein, H. Nutritional and lifestyle habits and water-fiber interaction in colorectal adenoma etiology. Am. Assoc. Cancer Res. 1997, 6, 79-85.

40. Martinez, M.E.; McPherson, R.S.; Annegers, J.F.; Levin, B. Association of diet and colorectal adenomatous polyps: Dietary fiber, calcium, and total fat. Epidemiology 1996, 7, 264-268. [CrossRef]

41. Mathew, A.; Peters, U.; Chatterjee, N.; Kulldorff, M.; Sinha, R. Fat, fiber, fruits, vegetables, and risk of colorectal adenomas. Int. J. Cancer 2004, 108, 287-292. [CrossRef] [PubMed]

42. Mujtaba, S.; Bostick, R.M. Differences in risk factor-colorectal adenoma associations according to non-steroidal anti-inflammatory drug use. Eur. J. Gastroenterol. Hepatol. 2018, 30, 1318-1326. [CrossRef]

43. Nimptsch, K.; Malik, V.S.; Fung, T.T.; Pischon, T.; Hu, F.B.; Willett, W.C.; Fuchs, C.S.; Ogino, S.; Chan, A.T.; Giovannucci, E.; et al. Dietary patterns during high school and risk of colorectal adenoma in a cohort of middle-aged women. Int. J. Cancer 2014, 134, 2458-2467. [CrossRef]

44. Peters, U.; Sinha, R.; Chatterjee, N.; Subar, A.F.; Ziegler, R.G.; Kulldorff, M.; Bresalier, R.; Weissfeld, J.L.; Flood, A.; Schatzkin, A.; et al. Dietary fibre and colorectal adenoma in a colorectal cancer early detection programme. Lancet 2003, 361, 1491-1495. [CrossRef]

45. Platz, E.A.; Giovannucci, E.; Rimm, E.B.; Rockett, H.R.; Stampfer, M.J.; Colditz, G.A.; Willett, W.C. Dietary fiber and distal colorectal adenoma in men. Cancer Epidemiol. Biomarkers Prev. 1997, 6, 661-670.

46. Sandler, R.S.; Lyles, C.M.; Peipins, L.A.; McAuliffe, C.A.; Woosley, J.T.; Kupper, L.L. Diet and risk of colorectal adenomas: Macronutrients, cholesterol, and fiber. J. Natl. Cancer Inst. 1993, 85, 884-891. [CrossRef]

47. Shaw, E.; Warkentin, M.T.; McGregor, S.E.; Town, S.; Hilsden, R.J.; Brenner, D.R. Intake of dietary fibre and lifetime non-steroidal anti-inflammatory drug (NSAID) use and the incidence of colorectal polyps in a population screened for colorectal cancer. J. Epidemiol. Community Health 2017, 71, 961-969. [CrossRef] [PubMed]

48. Tantamango, Y.M.; Knutsen, S.F.; Beeson, L.; Fraser, G.; Sabate, J. Association between dietary fiber and incident cases of colon polyps: The adventist health study. Gastrointest. Cancer Res. 2011, 4, 161-167.

49. Witte, J.S.; Longnecker, M.P.; Bird, C.L.; Lee, E.R.; Frankl, H.D.; Haile, R.W. Relation of vegetable, fruit, and grain consumption to colorectal adenomatous polyps. Am. J. Epidemiol. 1996, 144, 1015-1025. [CrossRef]

50. U.S. Department of Agriculture; U.S. Department of Health and Human Services. Dietary Guidelines for Americans, 2020-2025, 9th ed.; United States Department of Agriculture (USDA): Washington, DC, USA, 2020.

51. Angelino, D.; Godos, J.; Ghelfi, F.; Tieri, M.; Titta, L.; Lafranconi, A.; Marventano, S.; Alonzo, E.; Gambera, A.; Sciacca, S.; et al. Fruit and vegetable consumption and health outcomes: An umbrella review of observational studies. Int. J. Food Sci. Nutr. 2019, 70, 652-667. [CrossRef]

52. Gianfredi, V.; Vannini, S.; Moretti, M.; Villarini, M.; Bragazzi, N.L.; Izzotti, A.; Nucci, D. Sulforaphane and Epigallocatechin Gallate Restore Estrogen Receptor Expression by Modulating Epigenetic Events in the Breast Cancer Cell Line MDA-MB-231: A Systematic Review and Meta-Analysis. J. Nutr. 2017, 10, 126-135. [CrossRef]

53. Gianfredi, V.; Nucci, D.; Vannini, S.; Villarini, M.; Moretti, M. In vitro Biological Effects of Sulforaphane (SFN), Epigallocatechin-3gallate (EGCG), and Curcumin on Breast Cancer Cells: A Systematic Review of the Literature. Nutr. Cancer 2017, 69, 969-978. [CrossRef]

54. EFSA Panel on Dietetic Products Nutrition and Allergies (NDA). Scientific Opinion on Dietary Reference Values for carbohydrates and dietary fibre. EFSA J. 2010, 8, 77. [CrossRef]

55. Macfarlane, G.T.; Macfarlane, S. Bacteria, colonic fermentation, and gastrointestinal health. J. AOAC Int. 2012, 95, 50-60. [CrossRef]

56. Canani, R.B.; Costanzo, M.D.; Leone, L.; Pedata, M.; Meli, R.; Calignano, A. Potential beneficial effects of butyrate in intestinal and extraintestinal diseases. World J. Gastroenterol. 2011, 17, 1519-1528. [CrossRef] [PubMed]

57. Iebba, V.; Totino, V.; Gagliardi, A.; Santangelo, F.; Cacciotti, F.; Trancassini, M.; Mancini, C.; Cicerone, C.; Corazziari, E.; Pantanella, F.; et al. Eubiosis and dysbiosis: The two sides of the microbiota. New Microbiol. 2016, 39, 1-12.

58. Lobionda, S.; Sittipo, P.; Kwon, H.Y.; Lee, Y.K. The Role of Gut Microbiota in Intestinal Inflammation with Respect to Diet and Extrinsic Stressors. Microorganisms 2019, 7, 271. [CrossRef] [PubMed]

59. Ahn, J.; Sinha, R.; Pei, Z.; Dominianni, C.; Wu, J.; Shi, J.; Goedert, J.J.; Hayes, R.B.; Yang, L. Human gut microbiome and risk for colorectal cancer. J. Natl. Cancer Inst. 2013, 105, 1907-1911. [CrossRef]

60. FAO (Food and Agriculture Organization); WHO (World Health Organization). Carbohydrates in Human Nutrition; Report of a Joint FAO/WHO Expert Consultation (FAO Food and Nutrition Paper 66); Food and Agricolture Organization: Rome, Italy, 1998.

61. Williams, B.A.; Mikkelsen, D.; Flanagan, B.M.; Gidley, M.J. “Dietary fibre": Moving beyond the "soluble/insoluble" classification for monogastric nutrition, with an emphasis on humans and pigs. J. Anim. Sci. Biotechnol. 2019, 10, 45. [CrossRef] [PubMed]

62. World Cancer Research Fund; American Institute for Cancer Research. Diet., Nutrition, Physical Activity and Cancer: A Global Perspective; Continuous Update Project Expert Report 2018; World Cancer Research Fund: London, UK, 2018.

63. Bach-Faig, A.; Berry, E.M.; Lairon, D.; Reguant, J.; Trichopoulou, A.; Dernini, S.; Medina, F.X.; Battino, M.; Belahsen, R.; Miranda, G.; et al. Mediterranean diet pyramid today. Science and cultural updates. Public Health Nutr. 2011, 14, 2274-2284. [CrossRef] 
64. Fiolet, T.; Srour, B.; Sellem, L.; Kesse-Guyot, E.; Alles, B.; Mejean, C.; Deschasaux, M.; Fassier, P.; Latino-Martel, P.; Beslay, M.; et al. Consumption of ultra-processed foods and cancer risk: Results from NutriNet-Sante prospective cohort. BMJ Clin. Res. Ed. 2018, 360, k322. [CrossRef]

65. Monteiro, C.A.; Cannon, G.; Moubarac, J.C.; Levy, R.B.; Louzada, M.L.C.; Jaime, P.C. The UN Decade of Nutrition, the NOVA food classification and the trouble with ultra-processing. Public Health Nutr. 2018, 21, 5-17. [CrossRef] [PubMed]

66. Agus, A.; Denizot, J.; Thevenot, J.; Martinez-Medina, M.; Massier, S.; Sauvanet, P.; Bernalier-Donadille, A.; Denis, S.; Hofman, P.; Bonnet, R.; et al. Western diet induces a shift in microbiota composition enhancing susceptibility to Adherent-Invasive E. coli infection and intestinal inflammation. Sci. Rep. 2016, 6, 19032. [CrossRef]

67. Garcia-Montero, C.; Fraile-Martinez, O.; Gomez-Lahoz, A.M.; Pekarek, L.; Castellanos, A.J.; Noguerales-Fraguas, F.; Coca, S.; Guijarro, L.G.; Garcia-Honduvilla, N.; Asunsolo, A.; et al. Nutritional Components in Western Diet Versus Mediterranean Diet at the Gut Microbiota-Immune System Interplay. Implications for Health and Disease. Nutrients 2021, 13, 1579. [CrossRef]

68. Yu, G.; Bei, J.; Zhao, J.; Li, Q.; Cheng, C. Modification of carrot (Daucus carota Linn. var. Sativa Hoffm.) pomace insoluble dietary fiber with complex enzyme method, ultrafine comminution, and high hydrostatic pressure. Food Chem. 2018, 257, 333-340. [CrossRef]

69. Wang, Y.; Sun, P.; Li, H.; Adhikari, B.P.; Li, D. Rheological Behavior of Tomato Fiber Suspensions Produced by High Shear and High Pressure Homogenization and Their Application in Tomato Products. Int. J. Anal. Chem. 2018, 2018, 5081938. [CrossRef] [PubMed] 